\title{
Upper Estimates of the angle best approximations of generalized Liouville-Weyl derivatives
}

\begin{abstract}
In this article we consider continuous functions $f$ with period $2 \pi$ and their approximation by trigonometric polynomials. This article is devoted to the study of estimates of the best angular approximations of generalized Liouville-Weyl derivatives by angular approximation of functions in the three-dimensional case. We consider generalized Liouville-Weyl derivatives instead of the classical mixed Weyl derivative. In choosing the issues to be considered, we followed the general approach that emerged after the work of the second author of this article. Our main goal is to prove analogs of the results of in the three-dimensional case. The concept of general monotonic sequences plays a key role in our study. Several well-known inequalities are indicated for the norms, best approximations of the $r$-th derivative with respect to the best approximations of the function $f$. The issues considered in this paper are related to the range of issues studied in the works of Bernstein. Later Stechkin and Konyushkov obtained an inequality for the best approximation $f^{(r)}$. Also, in the works of Potapov, using the angle approximation, some classes of functions are considered. In subsection 1 we give the necessary notation and useful lemmas. Estimates for the norms and best approximations of the generalized Liouville-Weyl derivative in the three-dimensional case are obtained.
\end{abstract}

Keywords: Lebesgue space, best approximation by three-dimensional angle, trigonometric polynomial, Liouville-Weyl derivative.

\section{Introduction}

Let us mention several well-known inequalities for norms and best approximations of the $r$-th derivative in terms of best approximations of the function $f$.

The following result was proved by Bernstein for $p=\infty$ (for $1 \leq p<\infty$, see [2]) if $f \in L_{p}, 1 \leq p \leq \infty$, and $\sum_{k=0}^{\infty}(k+1)^{r-1} E_{k}(f)_{p}<\infty, r \in \mathbb{N}$, then $\left\|f^{(r)}\right\|_{p} \leq C(r) \sum_{k=0}^{\infty}(k+1)^{r-1} E_{k}(f)_{p}[1]$.

Later on, Stechkin [3] for $p=\infty$ and Konyushkov [4] for $1<p<\infty$ obtained the following inequality for the best approximations of $f^{(r)}$ :

$$
E_{n}\left(f^{(r)}\right)_{p} \leq C(r, p)\left(n^{r} E_{n}(f)_{p}+\sum_{k=n+1}^{\infty} k^{r-1} E_{k}(f)_{p}\right) \quad r, n \in \mathbb{N} .
$$

The last inequality was extended by the formula Timan [5] for the case of $1<p<\infty$ as follows:

$$
E_{n}\left(f^{(r)}\right)_{p} \leq C(r)\left(n^{r} E_{n}(f)_{p}+\left(\sum_{k=n+1}^{\infty} k^{\theta r-1} E_{k}^{\theta}(f)_{p}\right)^{\frac{1}{\theta}}\right), \quad \theta=\min (2, p) \quad r, n \in \mathbb{N} .
$$

Also, A. Jumabayeva and B. Simonov obtained estimates of norms and the angle best approximations of the generalized Liouville-Weyl derivatives by the angle approximation of functions in the two-dimensional case $[6,7]$.

Let $L_{p}\left(T^{3}\right), 1<p<\infty$ be the space of measurable functions of three variables that are $2 \pi$ periodic in each variable and such that

$$
\|f\|_{p}=\left(\int_{0}^{2 \pi} \int_{0}^{2 \pi} \int_{0}^{2 \pi}\left|f\left(x_{1}, x_{2}, x_{3}\right)\right|^{p} d x_{1} d x_{2} d x_{3}\right)^{1 / p}<\infty .
$$

${ }^{*}$ Corresponding author.

E-mail: akniet-1978@mail.ru 
$L_{p}^{0}$ is the set of functions $f \in L_{p}$ such that $\int_{0}^{2 \pi} f\left(x_{1}, x_{2}, x_{3}\right) d x_{1}=0$ for almost everyone $x_{2}, x_{3}$, $\int_{0}^{2 \pi} f\left(x_{1}, x_{2}, x_{3}\right) d x_{2}=0$ for almost everyone $x_{1}, x_{3}$ and $\int_{0}^{2 \pi} f\left(x_{1}, x_{2}, x_{3}\right) d x_{3}=0$ for almost everyone $x_{1}, x_{2}$.

Let $Y_{m_{1}, m_{2}, m_{3}}(f)_{p}$ be the best approximation by a three-dimensional angle of the function $f \in L_{p}\left(\mathbb{T}^{3}\right)$, i.e.

$$
Y_{m_{1}, m_{2}, m_{3}}(f)_{p}=\inf _{T_{m_{1}, \infty, \infty, T_{\infty}, m_{2}, \infty, T_{\infty}, \infty, m_{3}}}\left\|f-T_{m_{1}, \infty, \infty}-T_{\infty, m_{2}, \infty}-T_{\infty, \infty, m_{3}}\right\|_{p},
$$

where the function $T_{m_{1}, \infty, \infty}\left(x_{1}, x_{2}, x_{3}\right) \in L_{p}\left(\mathbb{T}^{3}\right)$ is a trigonometric polynomial of order at most $m_{1}$ in $x_{1}$, the function $T_{\infty, m_{2}, \infty}\left(x_{1}, x_{2}, x_{3}\right) \in L_{p}\left(\mathbb{T}^{3}\right)$ is a trigonometric polynomial of order at most $m_{2}$ in $x_{2}$ and the function $T_{\infty, \infty, m_{3}}\left(x_{1}, x_{2}, x_{3}\right) \in L_{p}\left(\mathbb{T}^{3}\right)$ is a trigonometric polynomial of order at most $m_{3}$ in $x_{3}$. In the work of Potapov using the angle approximation, some classes of functions are considered [8, 9].

By $\sigma(f)$ we denote the Fourier series of a function $f \in L_{p}\left(T^{3}\right)$, that is

$$
\sigma(f):=\sum_{k_{1}=-\infty}^{\infty} \sum_{k_{2}=-\infty}^{\infty} \sum_{k_{3}=-\infty}^{\infty} c_{k_{1}, k_{2}, k_{3}} e^{i\left(k_{1} x_{1}+k_{2} x_{2}+k_{3} x_{3}\right)}=\sum_{n_{1}=0}^{\infty} \sum_{n_{2}=0}^{\infty} \sum_{n_{3}=0}^{\infty} A_{n_{1}, n_{2}, n_{3}}\left(x_{1}, x_{2}, x_{3}\right),
$$

where $c_{k_{1}, k_{2}, k_{3}}=\frac{1}{8 \pi^{3}} \int_{0}^{2 \pi} \int_{0}^{2 \pi} \int_{0}^{2 \pi} f\left(x_{1}, x_{2}, x_{3}\right) e^{-i\left(k_{1} x_{1}+k_{2} x_{2}+k_{3} x_{3}\right)} d x_{1} d x_{2} d x_{3}$.

The transformed Fourier series of $\sigma(f)$ is given by

$$
\begin{gathered}
\sigma\left(f, \lambda, \beta_{1}, \beta_{2}, \beta_{3}\right)= \\
=\sum_{n_{1}=-\infty}^{\infty} \sum_{n_{2}=-\infty}^{\infty} \sum_{n_{3}=-\infty}^{\infty} \lambda_{n_{1}, n_{2}, n_{3}}\left[c_{n_{1} n_{2}, n_{3}} e^{i\left(n_{1} x_{1}+\beta_{1} \frac{\pi}{2}\right)} e^{i\left(n_{2} x_{2}+\beta_{2} \frac{\pi}{2}\right)} e^{i\left(n_{3} x_{3}+\beta_{3} \frac{\pi}{2}\right)}\left|n_{1}\right|^{\beta_{1}}\left|n_{2}\right|^{\beta_{2}}\left|n_{3}\right|^{\beta_{3}}\right],
\end{gathered}
$$

where $\beta_{1}, \beta_{2}, \beta_{3} \in R$ and $\lambda=\left\{\lambda_{n_{1}, n_{2}, n_{3}}\right\}_{n_{1} n_{2} n_{3} \in N}$ is a sequence of real numbers.

Let $\varphi\left(x_{1} x_{2} x_{3}\right) \sim \sigma\left(f, \lambda, \beta_{1}, \beta_{2}, \beta_{3}\right)$ is the $\left(\lambda, \beta_{1}, \beta_{2}, \beta_{3}\right)$ is the mixed derivative of the function $f$ (or Liouville-Weyl derivative) and denote it by $f^{\left(\lambda, \beta_{1}, \beta_{2}, \beta_{3}\right)}\left(x_{1} x_{2} x_{3}\right)$. For example, if $\lambda_{n_{1}, n_{2}, n_{3}}=n_{1}^{r_{1}} n_{2}^{r_{2}} n_{3}^{r_{3}}, r_{i}>0, \beta_{i}=r_{i}$ $(i=1,2, \ldots) \Rightarrow f^{\left(\lambda, \beta_{1}, \beta_{2}, \beta_{3}\right)}=f^{\left(r_{1}, r_{2}, r_{3}\right)}$, where $f^{\left(r_{1} r_{2} r_{3}\right)}$-mixed derivative of the function $f$ in the sense of Weyl.

Definition 1.1. [10, 11] A sequence $\lambda:=\left\{\lambda_{n}\right\}_{n=1}^{\infty}$ is said to be general monotone, written $\lambda \in G M^{3}$, if the relations

$$
\begin{aligned}
& \sum_{k_{1}=n_{1}}^{2 n_{1}}\left|\lambda_{k_{1}, n_{2}, n_{3}}-\lambda_{k_{1}+1, n_{2}, n_{3}}\right| \leq C\left|\lambda_{n_{1}, n_{2} n_{3},}\right|, \quad \sum_{k_{2}=n_{2}}^{2 n_{2}}\left|\lambda_{n_{1}, k_{2}, n_{3}}-\lambda_{n_{1} k_{2}+1, n_{3}}\right| \leq C\left|\lambda_{n_{1}, n_{2}, n_{3}}\right|, \\
& \sum_{k_{3}=n_{3}}^{2 n_{3}}\left|\lambda_{n_{1}, n_{2}, k_{3}}-\lambda_{n_{1}, n_{2}, k_{3}+1}\right| \leq C\left|\lambda_{n_{1}, n_{2}, n_{3},}\right| \\
& \sum_{k_{1}=n_{1}}^{2 n_{1}} \sum_{k_{2}=n_{2}}^{2 n_{2}}\left|\lambda_{k_{1}, k_{2}, n_{3}}-\lambda_{k_{1}+1, k_{2}, n_{3}}-\lambda_{k_{1}, k_{2}+1, n_{3}}+\lambda_{k_{1}+1, k_{2}+1, n_{3}}\right| \leq C\left|\lambda_{n_{1}, n_{2}, n_{3}}\right|, \\
& \sum_{k_{2}=n_{2}}^{2 n_{2}} \sum_{k_{3}=n_{3}}^{2 n_{3}}\left|\lambda_{n_{1}, k_{2}, k_{3}}-\lambda_{n_{1}, k_{2}+1, k_{3}}-\lambda_{n_{1}, k_{2}, k_{3}+1}+\lambda_{n_{1}, k_{2}+1, k_{3}}\right| \leq C\left|\lambda_{n_{1}, n_{2}, n_{3}}\right| \\
& \sum_{k_{1}=n_{1}}^{2 n_{1}} \sum_{k_{3}=n_{3}}^{2 n_{3}}\left|\lambda_{k_{1}, n_{2}, k_{3}}-\lambda_{k_{1}+1, n_{2}, k_{3}}-\lambda_{k_{1}, n_{2}, k_{3}+1}+\lambda_{k_{1}+1, n_{2}, k_{3}+1}\right| \leq C\left|\lambda_{n_{1}, n_{2}, n_{3}}\right| \text {, } \\
& \sum_{k_{1}=n_{1}}^{2 n_{1}} \sum_{k_{2}=n_{2}}^{2 n_{2}} \sum_{k_{3}=n_{3}}^{2 n_{3}} \mid \lambda_{k_{1}, k_{2}, k_{3}}-\lambda_{k_{1}+1, k_{2}, k_{3}}-\lambda_{k_{1}, k_{2}+1, k_{3}}-\lambda_{k_{1}, k_{2}, k_{3}+1}+ \\
& +\lambda_{k_{1}, k_{2}+1, k_{3}+1}+\lambda_{k_{1}+1, k_{2}, k_{3}+1}+\lambda_{k_{1}+1, k_{2}+1, k_{3}}-\lambda_{k_{1}+1, k_{2}+1, k_{3}+1}|\leq C| \lambda_{n_{1}, n_{2}, n_{3}} \mid
\end{aligned}
$$

hold for all integers $n_{1}, n_{2}$ and $n_{3}$, where the constant $C$ is independent of $n_{1}, n_{2}$ and $n_{3}$. 


\section{Auxiliary results}

In order to prove the main result, we formulate auxiliary statements. We denote

$$
\Delta_{m_{1}, m_{2}, m_{3}}:=\sum_{n_{1}=2^{m_{1}-1}}^{2^{m_{1}}-1} \sum_{n_{2}=2^{m_{2}-1}}^{2^{m_{2}}-1} \sum_{n_{3}=2^{m_{3}-1}}^{2^{m_{3}}-1} A_{n_{1}, n_{2}, n_{3}}\left(x_{1}, x_{2}, x_{3}\right), \quad m_{1}, m_{2}, m_{3}=1,2, \ldots
$$

Lemma 2.1. [11] $\left\{\lambda_{n}\right\} \in G M$ if and only if there exists $C>0$, such that

(i) $\left|\lambda_{k}\right| \leq C\left|\lambda_{n}\right|$ for $n \leq k \leq 2 n ;(i i) \sum_{k=n}^{N}\left|\Delta \lambda_{k}\right| \leq C\left(\left|\lambda_{n}\right|+\sum_{k=n+1}^{N} \frac{\left|\lambda_{k}\right|}{k}\right)$ for any $n<N$.

By [11], it follows that if $\left\{\lambda_{n_{1} n_{2} n_{3}}\right\} \in G M^{3}$, then

$$
\left|\lambda_{k_{1}, k_{2}, k_{3}}\right| \leq C\left|\lambda_{n_{1}, n_{2}, n_{3}}\right| \text { for } n_{1} \leq k_{1} \leq 2 n_{1}, n_{2} \leq k_{2} \leq 2 n_{2}, n_{3} \leq k_{3} \leq 2 n_{3} .
$$

This implies that the condition

$$
\begin{aligned}
& \sum_{k_{1}=n_{1}}^{2 n_{1}} \sum_{k_{2}=n_{2}}^{2 n_{2}} \sum_{k_{3}=n_{3}}^{2 n_{3}} \mid \lambda_{k_{1}, k_{2}, k_{3}}-\lambda_{k_{1}+1, k_{2}, k_{3}}-\lambda_{k_{1}, k_{2}+1, k_{3}}-\lambda_{k_{1}, k_{2}, k_{3}+1}+\lambda_{k_{1}, k_{2}+1, k_{3}+1}+ \\
& +\lambda_{k_{1}+1, k_{2}, k_{3}+1}+\lambda_{k_{1}+1, k_{2}+1, k_{3}}-\lambda_{k_{1}+1, k_{2}+1, k_{3}+1} \mid \leq C\left(\left|\lambda_{n_{1}, n_{2}, n_{3}}\right|+\left|\lambda_{2 n_{1}, 2 n_{2}, 2 n_{3}}\right|\right)
\end{aligned}
$$

is equivalent to the condition

$$
\begin{gathered}
\sum_{k_{1}=n_{1}}^{2 n_{1}} \sum_{k_{2}=n_{2}}^{2 n_{2}} \sum_{k_{3}=n_{3}}^{2 n_{3}} \mid \lambda_{k_{1}, k_{2}, k_{3}}-\lambda_{k_{1}+1, k_{2}, k_{3}}-\lambda_{k_{1}, k_{2}+1, k_{3}}-\lambda_{k_{1}, k_{2}, k_{3}+1}+ \\
+\lambda_{k_{1}, k_{2}+1, k_{3}+1}+\lambda_{k_{1}+1, k_{2}, k_{3}+1}+\lambda_{k_{1}+1, k_{2}+1, k_{3}}-\lambda_{k_{1}+1, k_{2}+1, k_{3}+1}|\leq C| \lambda_{n_{1}, n_{2}, n_{3}} \mid .
\end{gathered}
$$

Lemma 2.2. (Minkowskii inequality [12]) Let $1 \leq p<\infty$ and $a_{\nu k} \geq 0$, then

$$
\text { (a) }\left(\sum_{k=1}^{\infty}\left(\sum_{\nu=1}^{k} a_{\nu k}\right)^{p}\right)^{\frac{1}{p}} \leq \sum_{\nu=1}^{\infty}\left(\sum_{k=\nu}^{\infty} a_{\nu k}^{p}\right)^{\frac{1}{p}},(b)\left(\sum_{k=1}^{\infty}\left(\sum_{\nu=k}^{\infty} a_{\nu k}\right)^{p}\right)^{\frac{1}{p}} \leq \sum_{\nu=1}^{\infty}\left(\sum_{k=1}^{\nu} a_{\nu k}^{p}\right)^{\frac{1}{p}} .
$$

Lemma 2.3. [12] For a function $f(u, y)$ defined on measurable set $E=E_{1} \times E_{2} \subset \mathbb{R}_{n}$, where $x=(u, y)$, $u=\left(x_{1}, \ldots, x_{m}\right), y=\left(x_{m+1}, \ldots, x_{n}\right)$, the following inequality holds

$$
\left(\int_{E_{1}}\left|\int_{E_{2}} f(u, y) d y\right|^{p} d u\right)^{\frac{1}{p}} \leq \int_{E_{2}}\left(\int_{E_{1}}|f(u, y)|^{p} d u\right)^{\frac{1}{p}} d y .
$$

Lemma 2.4. [8] Let $f \in L_{p}\left(\mathbb{T}^{2}\right), 1<p<\infty, m_{i} \in \mathbb{N} \cup 0(i=1,2)$. Then

$$
\left\|f-S_{m_{1}, \infty}(f)-S_{\infty, m_{2}}(f)+S_{m_{1}, m_{2}}(f)\right\|_{p} \asymp Y_{m_{1}, m_{2}}(f)_{p},
$$

where $S_{m_{1}, m_{2}}$ are the partial sums of the Fourier series of a function $f$.

Lemma 2.5. [8] a) Let $1<p<\infty$ and (1) be the Fourier series of $f \in L_{p^{0}}\left(\mathbb{T}^{3}\right)$, then

$$
C_{1}(p)\|f\|_{p} \leq\left(\int_{0}^{2 \pi} \int_{0}^{2 \pi} \int_{0}^{2 \pi}\left(\sum_{m_{1}=1}^{\infty} \sum_{m_{2}=1}^{\infty} \sum_{m_{3}=1}^{\infty} \Delta_{m_{1} m_{2} m_{3}}^{2}\right)^{\frac{p}{2}} d x_{1} d x_{2} d x_{3}\right)^{\frac{1}{p}} \leq C_{2}(p)\|f\|_{p} .
$$

b) Let $1<p<\infty$. If (1) satisfies the following inequality

$$
I_{p}=\left(\int_{0}^{2 \pi} \int_{0}^{2 \pi} \int_{0}^{2 \pi}\left(\sum_{m_{1}=1}^{\infty} \sum_{m_{2}=1}^{\infty} \sum_{m_{3}=1}^{\infty} \Delta_{m_{1} m_{2} m_{3}}^{2}\right)^{\frac{p}{2}} d x_{1} d x_{2} d x_{3}\right)^{\frac{1}{p}}<\infty .
$$

Then (1) is the Fourier series of a function $f=\left(x_{1}, x_{2}, x_{3}\right) \in L_{p}\left(\mathbb{T}^{3}\right)$ and $\|f\|_{p} \leq C(p) I_{p}$. 


\section{Main result}

The aim of this paper is to prove the following theorem.

Theorem 3.1. Let $1<p<\infty, 0<\theta \leq \min (p, 2), \lambda:=\left\{\lambda_{n_{1}, n_{2}, n_{3}}\right\}_{n_{1}, n_{2}, n_{3}}$ be sequences of positive numbers such that $\lambda \in G M^{3}, \alpha_{i} \in \mathbb{R}_{+}, r_{i} \in \mathbb{R}_{+} \cup\{0\}$ and $\beta_{i} \in \mathbb{R}(i=1,2)$. If for $f \in L_{p}^{0}\left(\mathbb{T}^{3}\right)$ the series

$$
\begin{gathered}
\sum_{n_{1}=1}^{\infty}\left|\lambda_{n_{1}+1,1,1}^{\theta}-\lambda_{n_{1}, 1,1}^{\theta}\right| Y_{n_{1}, 0,0}^{\theta}(f)_{p}+ \\
+\sum_{n_{2}=1}^{\infty}\left|\lambda_{1, n_{2}+1,1}^{\theta}-\lambda_{1, n_{2}, 1}^{\theta}\right| Y_{0, n_{2}, 0}^{\theta}(f)_{p}+\sum_{n_{3}=1}^{\infty}\left|\lambda_{1,1, n_{3}+1}^{\theta}-\lambda_{1,1, n_{3}}^{\theta}\right| Y_{0,0, n_{3}}^{\theta}(f)_{p}+ \\
+\sum_{n_{1}=1}^{\infty} \sum_{n_{2}=1}^{\infty}\left|\lambda_{n_{1}+1, n_{2}+1,1}^{\theta}-\lambda_{n_{1}+1, n_{2}, 1}^{\theta}-\lambda_{n_{1}, n_{2}+1,1}^{\theta}+\lambda_{n_{1}, n_{2}, 1}^{\theta}\right| Y_{n_{1}, n_{2}, 0}^{\theta}(f)_{p}+ \\
+\sum_{n_{1}=1}^{\infty} \sum_{n_{3}=1}^{\infty}\left|\lambda_{n_{1}, 1, n_{3}}^{\theta}-\lambda_{n_{1}+1,1, n_{3}}^{\theta}-\lambda_{n_{1}, 1, n_{3}+1}^{\theta}+\lambda_{n_{1}+1,1, n_{3}+1}^{\theta}\right| Y_{n_{1}, 0, n_{3}}^{\theta}(f)_{p}+ \\
+\sum_{n_{2}=1}^{\infty} \sum_{n_{3}=1}^{\infty}\left|\lambda_{1, n_{2}, n_{3}}^{\theta}-\lambda_{1, n_{2}+1, n_{3}}^{\theta}-\lambda_{1, n_{2}, n_{3}+1}^{\theta}+\lambda_{1, n_{2}+1, n_{3}+1}^{\theta}\right| Y_{0, n_{2}, n_{3}}^{\theta}(f)_{p}+ \\
\quad+\sum_{n_{1}=1}^{\infty} \sum_{n_{2}=1}^{\infty} \sum_{n_{3}=1}^{\infty} \mid \lambda_{n_{1}, n_{2}, n_{3}}^{\theta}-\lambda_{n_{1}+1, n_{2}, n_{3}}^{\theta}-\lambda_{n_{1}, n_{2}+1, n_{3}}^{\theta}-\lambda_{n_{1}, n_{2}, n_{3}+1}^{\theta}+ \\
+\lambda_{n_{1}, n_{2}+1, n_{3}+1}^{\theta}+\lambda_{n_{1}+1, n_{2}, n_{3}+1}^{\theta}+\lambda_{n_{1}+1, n_{2}+1, n_{3}}^{\theta}-\lambda_{n_{1}+1, n_{2}+1, n_{3}+1}^{\theta} \mid Y_{n_{1}, n_{2}, n_{3}}^{\theta}(f)_{p}
\end{gathered}
$$

converges, then there exists a function $\varphi \in L_{p}^{0}\left(\mathbb{T}^{3}\right)$, with the Fourier series $\sigma\left(f, \lambda, \beta_{1}, \beta_{2}, \beta_{3}\right)$ and

$$
\begin{aligned}
& \|\varphi\|_{p} \leq\left(\lambda_{1,1,1}^{\theta}\|f\|_{p}^{\theta}+\sum_{n_{1}=1}^{\infty}\left|\lambda_{n_{1}+1,1,1}^{\theta}-\lambda_{n_{1}, 1,1}^{\theta}\right| Y_{n_{1}, 0,0}^{\theta}(f)_{p}+\right. \\
& +\sum_{n_{2}=1}^{\infty}\left|\lambda_{1, n_{2}+1,1}^{\theta}-\lambda_{1, n_{2}, 1}^{\theta}\right| Y_{0, n_{2}, 0}^{\theta}(f)_{p}+\sum_{n_{3}=1}^{\infty}\left|\lambda_{1,1, n_{3}+1}^{\theta}-\lambda_{1,1, n_{3}}^{\theta}\right| Y_{0,0, n_{3}}^{\theta}(f)_{p}+ \\
& +\sum_{n_{1}=1}^{\infty} \sum_{n_{2}=1}^{\infty}\left|\lambda_{n_{1}, n_{2}, 1}^{\theta}-\lambda_{n_{1}+1, n_{2}, 1}^{\theta}-\lambda_{n_{1}, n_{2}+1,1}^{\theta}+\lambda_{n_{1}+1, n_{2}+1,1}^{\theta}\right| Y_{n_{1}, n_{2}, 0}^{\theta}(f)_{p}+ \\
& +\sum_{n_{1}=1}^{\infty} \sum_{n_{3}=1}^{\infty}\left|\lambda_{n_{1}, 1, n_{3}}^{\theta}-\lambda_{n_{1}+1,1, n_{3}}^{\theta}-\lambda_{n_{1}, 1, n_{3}+1}^{\theta}+\lambda_{n_{1}+1,1, n_{3}+1}^{\theta}\right| Y_{n_{1}, 0, n_{3}}^{\theta}(f)_{p}+ \\
& +\sum_{n_{2}=1}^{\infty} \sum_{n_{3}=1}^{\infty}\left|\lambda_{1, n_{2}, n_{3}}^{\theta}-\lambda_{1, n_{2}+1, n_{3}}^{\theta}-\lambda_{1, n_{2}, n_{3}+1}^{\theta}+\lambda_{1, n_{2}+1, n_{3}+1}^{\theta}\right| Y_{0, n_{2}, n_{3}}^{\theta}(f)_{p}+ \\
& +\sum_{n_{1}=1}^{\infty} \sum_{n_{2}=1}^{\infty} \sum_{n_{3}=1}^{\infty} \mid \lambda_{n_{1}, n_{2}, n_{3}}^{\theta}-\lambda_{n_{1}+1, n_{2}, n_{3}}^{\theta}-\lambda_{n_{1}, n_{2}+1, n_{3}}^{\theta}-\lambda_{n_{1}, n_{2}, n_{3}+1}^{\theta}+ \\
& \left.+\lambda_{n_{1}, n_{2}+1, n_{3}+1}^{\theta}+\lambda_{n_{1}+1, n_{2}, n_{3}+1}^{\theta}+\lambda_{n_{1}+1, n_{2}+1, n_{3}}^{\theta}-\lambda_{n_{1}+1, n_{2}+1, n_{3}+1}^{\theta} \mid Y_{n_{1}, n_{2}, n_{3}}^{\theta}(f)_{p}\right)^{\frac{1}{\theta}},
\end{aligned}
$$

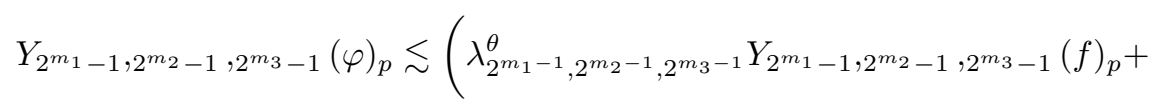

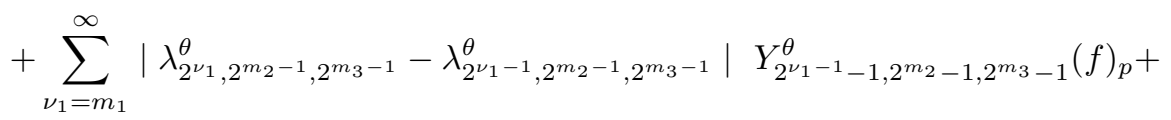




$$
\begin{aligned}
& +\sum_{\nu_{2}=m_{2}}^{\infty}\left|\lambda_{2^{m_{1}-1}, 2^{\nu_{2}, 2^{m_{3}-1}}}^{\theta}-\lambda_{2^{m_{1}-1}, 2^{\nu_{2}-1}, 2^{m_{3}-1}}^{\theta}\right| Y_{2^{m_{1}-1,2^{\nu_{2}-1}-1,2^{m_{3}-1}}}^{\theta}(f)_{p}+
\end{aligned}
$$

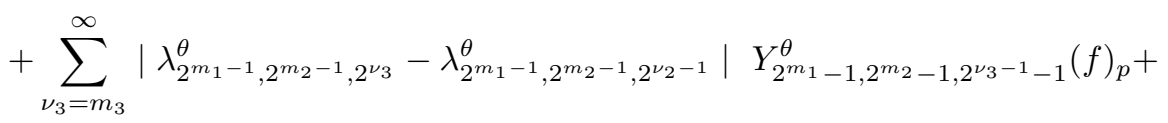

$$
\begin{aligned}
& +\sum_{\nu_{1}=m_{1}}^{\infty} \sum_{\nu_{2}=m_{2}}^{\infty} \mid \lambda_{2^{\nu_{1}, 2^{\nu_{2}}, 2^{m_{3}-1}}}^{\theta}-\lambda_{2^{\nu_{1}-1}, 2^{\nu_{2}, 2^{m_{3}-1}}-}^{\theta} \\
& -\lambda_{2^{\nu_{1}}, 2^{\nu_{2}-1}, 2^{m_{3}-1}}^{\theta}+\lambda_{2^{\nu_{1}-1}, 2^{\nu_{2}-1}, 2^{m_{3}-1}}^{\theta} \mid Y_{2^{\nu_{1}-1}-1,2^{\nu_{2}-1}-1,2^{m_{3}-1}}^{\theta}(f)_{p}+ \\
& +\sum_{\nu_{2}=m_{2}}^{\infty} \sum_{\nu_{3}=m_{3}}^{\infty} \mid \lambda_{2^{m_{1}-1}, 2^{\nu_{2}, 2^{\nu_{3}}}}^{\theta}-\lambda_{2^{m_{1}-1}, 2^{\nu_{2}-1}, 2^{\nu_{3}}-}^{\theta} \\
& -\lambda_{2^{m_{1}-1}, 2^{\nu_{2}}, 2^{\nu_{3}-1}}^{\theta}+\lambda_{2^{m_{1}-1}, 2^{\nu_{2}-1}, 2^{\nu_{3}-1}}^{\theta} \mid Y_{2^{m_{1}-1,2^{\nu_{2}-1}-1,2^{\nu_{3}-1}-1}}^{\theta}(f)_{p}+ \\
& +\sum_{\nu_{1}=m_{1}}^{\infty} \sum_{\nu_{3}=m_{3}}^{\infty} \mid \lambda_{2^{\nu_{1}}, 2^{m_{2}-1}, 2^{\nu_{3}}}^{\theta}-\lambda_{2^{\nu_{1}-1}, 2^{m_{2}-1}, 2^{\nu_{3}}-}^{\theta} \\
& -\lambda_{2^{\nu_{1}}, 2^{m_{2}-1}, 2^{\nu_{3}-1}}^{\theta}+\lambda_{2^{\nu_{1}-1}, 2^{m_{2}-1}, 2^{\nu_{3}-1}}^{\theta} \mid Y_{2^{\nu_{1}-1}-1,2^{m_{2}-1,2^{\nu_{3}-1}-1}}^{\theta}(f)_{p}+ \\
& +\sum_{\nu_{1}=m_{1}}^{\infty} \sum_{\nu_{2}=m_{2}}^{k_{2}} \sum_{\nu_{3}=m_{3}}^{\infty} \mid \lambda_{2^{\nu_{1}}, 2^{\nu_{2}, 2^{\nu_{3}}}}^{\theta}-\lambda_{2^{\nu_{1}-1}, 2^{\nu_{2}, 2^{\nu_{3}}}}^{\theta}-\lambda_{2^{\nu_{1}}, 2^{\nu_{2}-1}, 2^{\nu_{3}}}^{\theta}-\lambda_{2^{\nu_{1}}, 2^{\nu_{2}, 2^{\nu_{3}-1}}+}^{\theta}+ \\
& \left.+\lambda_{2^{\nu_{1}}, 2^{\nu_{2}-1}, 2^{\nu_{3}-1}}^{\theta}+\lambda_{2^{\nu_{1}-1}, 2^{\nu_{2}, 2^{\nu_{3}-1}}}^{\theta}+\lambda_{2^{\nu_{1}-1}, 2^{\nu_{2}-1}, 2^{\nu_{3}}}^{\theta}-\lambda_{2^{\nu_{1}-1}, 2^{\nu_{2}-1}, 2^{\nu_{3}-1}}^{\theta} \mid Y_{2^{\nu_{1}-1}-1,2^{\nu_{2}-1}-1,2^{\nu_{3}-1}-1}^{\theta}(f)_{p}\right)^{\frac{1}{\theta}} .
\end{aligned}
$$

Proof. Let the series (2) be convergent and $f \in L_{p}^{0}\left(T^{3}\right)$. We use the following inequality

$$
\begin{gathered}
\lambda_{2^{n_{1}-1}, 2^{n_{2}-1}, 2^{n_{3}-1}}^{\theta} \leq \lambda_{111}^{\theta}+\sum_{m_{3}=2}^{n_{3}}\left|\lambda_{1,1,2^{m_{3}-1}}^{\theta}-\lambda_{1,1,2^{m_{3}-2}}^{\theta}\right|+ \\
+\sum_{m_{2}=2}^{n_{2}}\left|\lambda_{1,2^{m_{2}-1}, 1}^{\theta}-\lambda_{1,2^{m_{2}-2}, 1}^{\theta}\right|+\sum_{m_{1}=2}^{n_{1}}\left|\lambda_{2^{m_{1}-1}, 1,1}^{\theta}-\lambda_{2^{m_{1}-2}, 1,1}^{\theta}\right|+ \\
+\sum_{m_{1}=2}^{n_{1}} \sum_{m_{2}=2}^{n_{2}}\left|\lambda_{2^{m_{1}-1}, 2^{m_{2}-1}, 1}^{\theta}-\lambda_{2^{m_{1}-2}, 2^{m_{2}-1}, 1}^{\theta}-\lambda_{2^{m_{1}-1}, 2^{m_{2}-2}, 1}^{\theta}+\lambda_{2^{m_{1}-2}, 2^{m_{2}-2}, 1}^{\theta}\right|+ \\
+\sum_{m_{2}=2}^{n_{2}} \sum_{m_{3}=2}^{n_{3}}\left|\lambda_{1,2^{m_{2}-1}, 2^{m_{3}-1}}^{\theta}-\lambda_{1,2^{m_{2}-2}, 2^{m_{3}-1}}^{\theta}-\lambda_{1,2^{m_{2}-1}, 2^{m_{3}-2}}^{\theta}+\lambda_{1,2^{m_{2}-2}, 2^{m_{3}-2}}^{\theta}\right|+ \\
+\sum_{m_{1}=2}^{n_{1}} \sum_{m_{3}=2}^{n_{3}}\left|\lambda_{2^{m_{1}-1}, 1,2^{m_{3}-1}}^{\theta}-\lambda_{2^{m_{1}-2}, 1,2^{m_{3}-1}}^{\theta}-\lambda_{2^{m_{1}-1}, 1,2^{m_{3}-2}}^{\theta}+\lambda_{2^{m_{1}-2}, 1,2^{m_{3}-2}}^{\theta}\right|+ \\
+\sum_{m_{1}=2}^{n_{1}} \sum_{m_{2}=2}^{n_{2}} \sum_{m_{3}=2}^{n_{3}} \mid \lambda_{2^{m_{1}-1}, 2^{m_{2}-1}, 2^{m_{3}-1}}^{\theta}-\lambda_{2^{m_{1}-1}, 2^{m_{2}-2}, 2^{m_{3}-2}}^{\theta}-\lambda_{2^{m_{1}-2}, 2^{m_{2}-1}, 2^{m_{3}-2}}^{\theta}- \\
-\lambda_{2^{m_{1}-2}, 2^{m_{2}-2}, 2^{m_{3}-1}}^{\theta}-\lambda_{2^{m_{1}-1}, 2^{m_{2}-1}, 2^{m_{3}-2}}^{\theta}+\lambda_{2^{m_{1}-2}, 2^{m_{2}-1}, 2^{m_{3}-1}}^{\theta}-\lambda_{2^{m_{1}-1}, 2^{m_{2}-2}, 2^{m_{3}-1}}^{\theta}+ \\
+\lambda_{2^{m_{1}-2}, 2^{m_{2}-2}, 2^{m_{3}-2}}^{\theta} \mid .
\end{gathered}
$$

Let us denote $\Delta_{n_{1}, n_{2}, n_{3}}=\sum_{\nu_{1}=2^{n_{1}-1}}^{2^{n_{1}}-1} \sum_{\nu_{2}=2^{n_{2}-1}}^{2^{n_{2}}-1} \sum_{\nu_{3}=2^{n_{3}-1}}^{2^{n_{3}}-1} A_{\nu_{1}, \nu_{2}, \nu_{3}}\left(f, x_{1}, x_{2}, x_{3}\right)\left(n_{1}, n_{2}, n_{3}=1,2 \ldots\right)$.

Using (5) and property of $G M$ (Lemma 2.1), we get

$$
I_{1}=\left\{\int_{0}^{2 \pi} \int_{0}^{2 \pi} \int_{0}^{2 \pi}\left[\sum_{n_{1}=1}^{\infty} \sum_{n_{2}=1}^{\infty} \sum_{n_{3}=1}^{\infty} \lambda_{2^{n_{1}-1}, 2^{n_{2}-1}, 2^{n_{3}-1}}^{2} \Delta_{n_{1}, n_{2}, n_{3}}^{2}\right]^{\frac{p}{2}} d x_{1}, d x_{2}, d x_{3}\right\}^{\frac{1}{p}}=
$$




$$
\begin{aligned}
& =\left\|\left[\sum_{n_{1}=1}^{\infty} \sum_{n_{2}=1}^{\infty} \sum_{n_{3}=1}^{\infty} \lambda_{2^{n_{1}-1}, 2^{n_{2}-1}, 2^{n_{3}-1}}^{2} \Delta_{n_{1}, n_{2}, n_{3}}^{2}\right]^{\frac{1}{2}}\right\|_{p}= \\
& =\|\left[\lambda_{1,1,1}^{2} \Delta_{1,1,1}^{2}+\sum_{n_{1}=2}^{\infty} \lambda_{2^{n_{1}-1}, 1,1}^{2} \Delta_{n_{1}, 1,1}^{2}+\sum_{n_{2}=2}^{\infty} \lambda_{1,2^{n_{2}-1}, 1}^{2} \Delta_{1, n_{2}, 1}^{2}+\sum_{n_{3}=2}^{\infty} \lambda_{1,1,2^{n_{3}-1}}^{2} \Delta_{1,1, n^{3}}^{2}+\right. \\
& +\sum_{n_{1}=2}^{\infty} \sum_{n_{2}=2}^{\infty} \lambda_{2^{n_{1}-1}, 2^{n_{2}-1}, 1}^{2} \Delta_{n_{1}, n_{2}, 1}^{2}+\sum_{n_{1}=2}^{\infty} \sum_{n_{3}=2}^{\infty} \lambda_{2^{n_{1}-1}, 1,2^{n_{3}-1}}^{2} \Delta_{n_{1}, 1, n_{3}}^{2}+ \\
& \left.+\sum_{n_{2}=2}^{\infty} \sum_{n_{3}=2}^{\infty} \lambda_{1,2^{n_{2}-1}, 2^{n_{3}-1}}^{2} \Delta_{1, n_{2}, n_{3}}^{2}+\sum_{n_{1}=2}^{\infty} \sum_{n_{2}=2}^{\infty} \sum_{n_{3}=2}^{\infty} \lambda_{2^{n_{1}-1}, 2^{n_{2}-1}, 2^{n_{3}-1}}^{2} \Delta_{n_{1}, n_{2}, n_{2}}^{2}\right]^{\frac{1}{2}} \|_{p} \lesssim \\
& \lesssim \lambda_{1,1,1}\left\|\left[\sum_{n_{1}=1}^{\infty} \sum_{n_{2}=1}^{\infty} \sum_{n_{3}=1}^{\infty} \Delta_{n_{1}, n_{2}, n_{3}}^{2}\right]^{\frac{1}{2}}\right\|_{p}+\left\|\left(\sum_{n_{1}=2}^{\infty} \sum_{n_{2}=1}^{\infty} \sum_{n_{3}=1}^{\infty} \Delta_{n_{1}, n_{2}, n_{3}}^{2}\left[\sum_{\nu_{1}=2}^{n_{1}}\left|\lambda_{2^{\nu_{1}-1,1,1}}^{\theta}-\lambda_{2^{\nu_{1}-2}, 1,1}^{\theta}\right|\right]^{\frac{2}{\theta}}\right)^{\frac{1}{2}}\right\|_{p}+ \\
& +\left\|\left(\sum_{n_{1}=1}^{\infty} \sum_{n_{2}=2}^{\infty} \sum_{n_{3}=1}^{\infty} \Delta_{n_{1}, n_{2}, n_{3}}^{2}\left[\sum_{\nu_{2}=2}^{n_{2}}\left|\lambda_{1,2^{\nu_{2}-1}, 1}^{\theta}-\lambda_{1,2^{\nu_{2}-2}, 1}^{\theta}\right|\right]^{\frac{2}{\theta}}\right)^{\frac{1}{2}}\right\|_{p}+ \\
& +\left\|\left(\sum_{n_{1}=1}^{\infty} \sum_{n_{2}=1}^{\infty} \sum_{n_{3}=2}^{\infty} \Delta_{n_{1}, n_{2}, n_{3}}^{2}\left[\sum_{\nu_{3}=2}^{n_{2}}\left|\lambda_{1,1,2^{\nu_{3}-1}}^{\theta}-\lambda_{1,1,2^{\nu_{3}-2}}^{\theta}\right|\right]^{\frac{2}{\theta}}\right)^{\frac{1}{2}}\right\|_{p}+ \\
& +\|\left(\sum _ { n _ { 1 } = 2 } ^ { \infty } \sum _ { n _ { 2 } = 2 } ^ { \infty } \sum _ { n _ { 3 } = 1 } ^ { \infty } \Delta _ { n _ { 1 } , n _ { 2 } , n _ { 3 } } ^ { 2 } \left(\sum_{\nu_{1}=2}^{n_{1}} \sum_{\nu_{2}=2}^{n_{2}} \mid \lambda_{2^{\nu_{1}-1}, 2^{\nu_{2}-1}, 1}^{\theta}-\lambda_{2^{\nu_{1}-2}, 2^{\nu_{2}-1}, 1}^{\theta}-\lambda_{2^{\nu_{1}-1}, 2^{\nu_{2}-2}, 1}^{\theta}+\right.\right. \\
& \left.\left.+\lambda_{2^{\nu_{1}-2}, 2^{\nu_{2}-2}, 1}^{\theta} \mid\right)^{\frac{2}{\theta}}\right)^{\frac{1}{2}}\left\|_{p}+\right\|\left(\sum _ { n _ { 1 } = 2 } ^ { \infty } \sum _ { n _ { 2 } = 1 } ^ { \infty } \sum _ { n _ { 3 } = 2 } ^ { \infty } \Delta _ { n _ { 1 } , n _ { 2 } , n _ { 3 } } ^ { 2 } \left(\sum_{\nu_{1}=2}^{n_{1}} \sum_{\nu_{3}=2}^{n_{3}} \mid \lambda_{2^{\nu_{1}-1}, 1,2^{\nu_{3}-1}}^{\theta}-\lambda_{2^{\nu_{1}-2}, 1,2^{\nu_{3}-1}}^{\theta}-\right.\right. \\
& \left.\left.-\lambda_{2^{\nu_{1}-1}, 1,2^{\nu_{3}-2}}^{\theta}+\lambda_{2^{\nu_{1}-2}, 1,2^{\nu_{3}-2}}^{\theta} \mid\right)^{\frac{2}{\theta}}\right)^{\frac{1}{2}}\left\|_{p}+\right\|\left(\sum _ { n _ { 1 } = 1 } ^ { \infty } \sum _ { n _ { 2 } = 2 } ^ { \infty } \sum _ { n _ { 3 } = 2 } ^ { \infty } \Delta _ { n _ { 1 } , n _ { 2 } , n _ { 3 } } ^ { 2 } \left(\sum_{\nu_{2}=2}^{n_{2}} \sum_{\nu_{3}=2}^{n_{3}} \mid \lambda_{1,2^{\nu_{2}-1}, 2^{\nu_{3}-1}}^{\theta}-\right.\right. \\
& \left.\left.-\lambda_{1,2^{\nu_{2}-2}, 2^{\nu_{3}-1}}^{\theta}-\lambda_{1,2^{\nu_{2}-1}, 2^{\nu_{3}-2}}^{\theta}+\lambda_{1,2^{\nu_{2}-2}, 2^{\nu_{3}-2}}^{\theta} \mid\right)^{\frac{2}{\theta}}\right)^{\frac{1}{2}} \|_{p}+ \\
& +\|\left(\sum _ { n _ { 1 } = 2 } ^ { \infty } \sum _ { n _ { 2 } = 2 } ^ { \infty } \sum _ { n _ { 3 } = 2 } ^ { \infty } \Delta _ { n _ { 1 } , n _ { 2 } , n _ { 3 } } ^ { 2 } \left[\sum_{\nu_{1}=2}^{n_{1}} \sum_{\nu_{2}=2}^{n_{2}} \sum_{\nu_{3}=2}^{n_{3}} \mid \lambda_{2^{\nu_{1}-1}, 2^{\nu_{2}-1}, 2^{\nu_{3}-1}}^{\theta}-\lambda_{2^{\nu_{1}-1}, 2^{\nu_{2}-1}, 2^{\nu_{3}-2}-}^{\theta}\right.\right.
\end{aligned}
$$

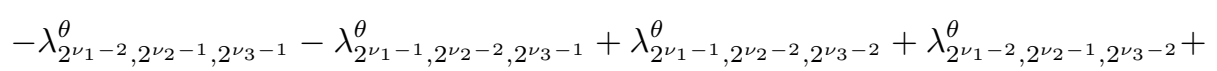

$$
\begin{aligned}
& \left.\left.+\lambda_{2^{\nu_{1}-2}, 2^{\nu_{2}-2}, 2^{\nu_{3}-1}}^{\theta}-\lambda_{2^{\nu_{1}-2}, 2^{\nu_{2}-2}, 2^{\nu_{3}-2}}^{\theta} \mid\right]^{\frac{2}{\theta}}\right)^{\frac{1}{2}} \|_{p}=: H_{1}+H_{2}+H_{3}+H_{4}+H_{5}+H_{6}+H_{7}+H_{8} .
\end{aligned}
$$

Let us estimate $H_{1}$. Applying Lemma 2.5, we have $H_{1} \leq C \lambda_{1,1,1}\|f\|_{p}<\infty$. Now we estimate $H_{2}$ :

$$
H_{2}=\left\{\int_{0}^{2 \pi} \int_{0}^{2 \pi} \int_{0}^{2 \pi}\left[\sum_{n_{1}=2}^{\infty} \sum_{n_{2}=1}^{\infty} \sum_{n_{3}=1}^{\infty} \Delta_{n_{1}, n_{2}, n_{3}}^{2}\left[\sum_{\nu_{1}=2}^{n_{1}}\left|\lambda_{2^{\nu_{1}-1}, 1,1}^{\theta}-\lambda_{2^{\nu_{1}-2}, 1,1}^{\theta}\right|\right]^{\frac{2}{\theta}}\right]^{\frac{p}{2}} d x_{1}, d x_{2}, d x_{3}\right\}^{\frac{1}{p}} .
$$

Using Minkowski's inequality and Lemma 2.2 (a) for $\frac{2}{\theta} \geq 1$, we derive

$$
\begin{gathered}
\sum_{n_{3}=1}^{\infty} \sum_{n_{2}=1}^{\infty} \sum_{n_{1}=2}^{\infty} \Delta_{n_{1}, n_{2}, n_{3}}^{2}\left[\sum_{\nu_{1}=2}^{n_{1}}\left|\lambda_{2^{\nu_{1}-1,1,1}}^{\theta}-\lambda_{2^{\nu_{1}-2}, 1,1}^{\theta}\right|\right]^{\frac{2}{\theta}}= \\
=\sum_{n_{1}=2}^{\infty}\left(\left(\sum_{n_{2}=1}^{\infty} \sum_{n_{3}=1}^{\infty}\left[\sum_{\nu_{1}=2}^{n_{1}}\left|\Delta_{n_{1}, n_{2}, n_{3}}\right| \theta\left|\lambda_{2^{\nu_{1}-1}, 1,1}^{\theta}-\lambda_{2^{\nu_{1}-2}, 1,1}^{\theta}\right|\right]^{\frac{2}{\theta}}\right)^{\frac{\theta}{2}} \leq\right.
\end{gathered}
$$

Mathematics series. № 3(103)/2021 


$$
\begin{gathered}
\leq \sum_{n_{1}=2}^{\infty}\left(\sum_{\nu_{1}=2}^{n_{1}}\left[\sum_{n_{2}=1}^{\infty} \sum_{n_{3}=1}^{\infty}\left|\Delta_{n_{1}, n_{2}, n_{3}}\right|^{2}\left|\lambda_{2^{\nu_{1}-1}, 1,1}^{\theta}-\lambda_{2^{\nu_{1}-2}, 1,1}^{\theta}\right|^{\frac{2}{\theta}}\right]^{\frac{\theta}{2}}=\right. \\
=\left(\left(\sum_{n_{1}=2}^{\infty}\left\{\sum_{\nu_{1}=2}^{n_{1}}\left[\sum_{n_{2}=1}^{\infty} \sum_{n_{3}=1}^{\infty}\left|\Delta_{n_{1}, n_{2}, n_{3}}\right|^{2}\left|\lambda_{2^{\nu_{1}-1}, 1,1}^{\theta}-\lambda_{2^{\nu_{1}-2}, 1,1}^{\theta}\right|^{\frac{2}{\theta}}\right]^{\frac{\theta}{2}}\right\}^{\frac{2}{\theta}}\right)^{\frac{\theta}{2}} \leq\right. \\
\leq\left(\sum_{\nu_{1}=2}^{\infty}\left\{\sum_{n_{1}=\nu_{1}}^{\infty} \sum_{n_{2}=1}^{\infty} \sum_{n_{3}=1}^{\infty}\left|\Delta_{n_{1}, n_{2}, n_{3}}\right|^{2}\left|\lambda_{2^{\nu_{1}-1}, 1,1}^{\theta}-\lambda_{2^{\nu_{1}-2}, 1,1}^{\theta}\right|^{\frac{2}{\theta}}\right\}^{\frac{\theta}{2}}\right)^{\frac{2}{\theta}}= \\
=\left(\sum_{\nu_{1}=2}^{\infty}\left|\lambda_{2^{\nu_{1}-1}, 1,1}^{\theta}-\lambda_{2^{\nu_{1}-2}, 1,1}^{\theta}\right|\left(\sum_{n_{1}=\nu_{1}}^{\infty} \sum_{n_{2}=1}^{\infty} \sum_{n_{3}=1}^{\infty}\left|\Delta_{n_{1}, n_{2} n_{3}}\right|^{2}\right)^{\frac{\theta}{2}}\right)^{\frac{2}{\theta}} .
\end{gathered}
$$

Applying this inequality, we obtain

$$
\begin{aligned}
H_{2} & \leq\left\{\int_{0}^{2 \pi} \int_{0}^{2 \pi} \int_{0}^{2 \pi}\left[\left\{\sum_{\nu_{1}=2}^{\infty}\left|\lambda_{2^{\nu_{1}-1}, 1,1}^{\theta}-\lambda_{2^{\nu_{1}-2}, 1,1}^{\theta}\right|\left(\sum_{n_{1}=\nu_{1}}^{\infty} \sum_{n_{2}=1}^{\infty} \sum_{n_{3}=1}^{\infty}\left|\Delta_{n_{1}, n_{2} n_{3}}\right|^{2}\right)^{\frac{\theta}{2}}\right\}^{\frac{2}{\theta}}\right]^{\frac{p}{2}} d x_{1}, d x_{2}, d x_{3}\right\}^{\frac{1}{p}}= \\
& =\left(\left\{\int_{0}^{2 \pi} \int_{0}^{2 \pi} \int_{0}^{2 \pi}\left(\sum_{\nu_{1}=2}^{\infty}\left|\lambda_{2^{\nu_{1}-1,1,1}}^{\theta}-\lambda_{2^{\nu_{1}-2,1,1}}^{\theta}\right|\left(\sum_{n_{1}=\nu_{1}}^{\infty} \sum_{n_{2}=1}^{\infty} \sum_{n_{3}=1}^{\infty}\left|\Delta_{n_{1}, n_{2}, n_{3}}\right|^{2}\right)^{\frac{\theta}{2}}\right)^{\frac{p}{\theta}} d x_{1}, d x_{2}, d x_{3}\right\}^{\frac{\theta}{p}}\right)^{\frac{1}{\theta}} .
\end{aligned}
$$

Further, using Minkowski's inequality for $\frac{p}{\theta} \geq 1$, Lemmas 2.4 and 2.5, we have

$$
\begin{gathered}
H_{2} \leq\left(\sum_{\nu_{1}=2}^{\infty}\left|\lambda_{2^{\nu_{1}-1}, 1,1}^{\theta}-\lambda_{2^{\nu_{1}-2}, 1,1}^{\theta}\right|\left\{\int_{0}^{2 \pi} \int_{0}^{2 \pi} \int_{0}^{2 \pi}\left(\left(\sum_{n_{1}=\nu_{1}}^{\infty} \sum_{n_{2}=1}^{\infty} \sum_{n_{3}=1}^{\infty}\left|\Delta_{n_{1}, n_{2}, n_{3}}\right|^{2}\right)^{\frac{\theta}{2}}\right)^{\frac{p}{\theta}} d x_{1}, d x_{2}, d x_{3}\right\}^{\frac{\theta}{p}}\right)^{\frac{1}{\theta}}= \\
=\left(\sum_{\nu_{1}=2}^{\infty}\left|\lambda_{2^{\nu_{1}-1}, 1,1}^{\theta}-\lambda_{2^{\nu_{1}-2}, 1,1}^{\theta}\right|\left\|\left(\sum_{n_{1}=\nu_{1}}^{\infty} \sum_{n_{2}=1}^{\infty} \sum_{n_{3}=1}^{\infty}\left|\Delta_{n_{1}, n_{2}, n_{3}}\right|^{2}\right)^{\frac{1}{2}}\right\|_{p}^{\theta}\right)^{\frac{1}{\theta}} \lesssim \\
\lesssim\left(\sum_{\nu_{1}=2}^{\infty}\left|\lambda_{2^{\nu_{1}-1}, 1,1}^{\theta}-\lambda_{2^{\nu_{1}-1}, 1,1}^{\theta}\right| Y_{2^{\nu_{1}}-1,0,0}^{\theta}(f)_{p}\right)^{\frac{1}{\theta}} .
\end{gathered}
$$

Thus, we obtain $H_{2} \lesssim\left(\sum_{\nu_{1}=2}^{\infty}\left|\lambda_{2^{\nu_{1}}, 1,1}^{\theta}-\lambda_{2^{\nu_{1}-1}, 1,1}^{\theta}\right| Y_{2^{\nu_{1}}-1,0,0}^{\theta}(f)_{p}\right)^{\frac{1}{\theta}}$. From (2) it follows that $H_{2}<\infty$, $H_{3}, H_{4}$ can be estimated similarly to $H_{2}$ and we have

$$
H_{3} \lesssim\left(\sum_{\nu_{2}=1}^{\infty}\left|\lambda_{1,2^{\nu_{2}, 1}}^{\theta}-\lambda_{1,2^{\nu_{2}-1}, 1}^{\theta}\right| Y_{0,2^{\nu_{2}-1,0}}^{\theta}(f)_{p}\right)^{\frac{1}{\theta}}, H_{4} \lesssim\left(\sum_{\nu_{3}=1}^{\infty}\left|\lambda_{1,1,2^{\nu_{2}}}^{\theta}-\lambda_{1,1,2^{\nu_{2}-1}}^{\theta}\right| Y_{0,0,2^{\nu_{3}-1}}^{\theta}(f)_{p}\right)^{\frac{1}{\theta}} .
$$

To estimate $H_{5}$, we apply the method of estimate for $H_{4}$ as in article [9]. First, we obtain the upper estimate of the following sum. Applying Lemmas 2.2 and 2.3 twice for $\frac{2}{\theta} \geq 1$, we get

$$
\begin{aligned}
& \sum_{n_{3}=1}^{\infty} \sum_{n_{2}=2}^{\infty} \sum_{n_{1}=2}^{\infty} \Delta_{n_{1}, n_{2}, n_{3}}^{2}\left[\sum_{\nu_{1}=2}^{n_{1}} \sum_{\nu_{2}=2}^{n_{2}}\left|\lambda_{2^{\nu_{1}-1}, 2^{\nu_{2}-1}, 1}^{\theta}-\lambda_{2^{\nu_{1}-2}, 2^{\nu_{2}-1}, 1}^{\theta}-\lambda_{2^{\nu_{1}-1}, 2^{\nu_{2}-2}, 1}^{\theta}+\lambda_{2^{\nu_{1}-2}, 2^{\nu_{2}-2}, 1}^{\theta}\right|\right]^{2 / \theta} \leq \\
\leq & \left(\sum_{\nu_{2}=2}^{\infty} \sum_{\nu_{1}=2}^{\infty}\left|\lambda_{2^{\nu_{1}-1}, 2^{\nu_{2}-1}, 1}^{\theta}-\lambda_{2^{\nu_{1}-2}, 2^{\nu_{2}-1}, 1}^{\theta}-\lambda_{2^{\nu_{1}-1}, 2^{\nu_{2}-2}, 1}^{\theta}+\lambda_{2^{\nu_{1}-2}, 2^{\nu_{2}-2}, 1}^{\theta}\right|\left(\sum_{n_{1}=\nu_{1}}^{\infty} \sum_{n_{2}=\nu_{2}}^{\infty} \sum_{n_{3}=1}^{\infty}\left|\Delta_{n_{1}, n_{2}, n_{3}}\right|^{2}\right)^{\frac{\theta}{2}}\right)^{\frac{2}{\theta}} .
\end{aligned}
$$

Hence, Lemma 2.3 with $\frac{p}{\theta} \geq 1$ implies that

$$
H_{5} \leq\left(\left\{\int _ { 0 } ^ { 2 \pi } \int _ { 0 } ^ { 2 \pi } \int _ { 0 } ^ { 2 \pi } \left[\sum_{\nu_{2}=2}^{\infty} \sum_{\nu_{1}=2}^{\infty} \mid \lambda_{2^{\nu_{1}-1}, 2^{\nu_{2}-1}, 1}^{\theta}-\lambda_{2^{\nu_{1}-2}, 2^{\nu_{2}-1}, 1}^{\theta}\right.\right.\right.
$$




$$
\begin{aligned}
& \left.\left.\left.-\lambda_{2^{\nu_{1}-1}, 2^{\nu_{2}-2}, 1}^{\theta}+\lambda_{2^{\nu_{1}-2}, 2^{\nu_{2}-2}, 1}^{\theta} \mid\left(\sum_{n_{1}=\nu_{1}}^{\infty} \sum_{n_{2}=\nu_{2}}^{\infty} \sum_{n_{3}=1}^{\infty}\left|\Delta_{n_{1}, n_{2}, n_{3}}\right|^{2}\right)^{\frac{\theta}{2}}\right]^{\frac{p}{\theta}} d x_{1}, d x_{2}, d x_{3}\right\}^{\frac{\theta}{p}}\right)^{\frac{1}{\theta}} \leq \\
& \leq\left(\sum_{\nu_{2}=2}^{\infty} \sum_{\nu_{1}=2}^{\infty} \mid \lambda_{2^{\nu_{1}-1}, 2^{\nu_{2}-1}, 1}^{\theta}-\lambda_{2^{\nu_{1}-2}, 2^{\nu_{2}-1}, 1}^{\theta}-\lambda_{2^{\nu_{1}-1}, 2^{\nu_{2}-2}, 1}^{\theta}+\right. \\
& \left.+\lambda_{2^{\nu_{1}-2}, 2^{\nu_{2}-2}, 1}^{\theta}\left\{\int_{0}^{2 \pi} \int_{0}^{2 \pi} \int_{0}^{2 \pi}\left[\sum_{n_{1}=\nu_{1}}^{\infty} \sum_{n_{2}=\nu_{2}}^{\infty} \sum_{n_{3}=1}^{\infty}\left|\Delta_{n_{1}, n_{2}, n_{3}}\right|^{2}\right]^{\frac{p}{\theta}} d x_{1}, d x_{2}, d x_{3}\right\}^{\frac{\theta}{p}}\right)^{\frac{1}{\theta}} .
\end{aligned}
$$

By Lemmas 2.4 and 2.5, we obtain

$$
H_{5} \lesssim\left(\sum_{\nu_{2}=1}^{\infty} \sum_{\nu_{1}=1}^{\infty}\left|\lambda_{2^{\nu_{1}}, 2^{\nu_{2}, 1}}^{\theta}-\lambda_{2^{\nu_{1}-1}, 2^{\nu_{2}, 1}}^{\theta}-\lambda_{2^{\nu_{1}, 2^{\nu_{2}-1}, 1}}^{\theta}+\lambda_{2^{\nu_{1}-1}, 2^{\nu_{2}-1}, 1}^{\theta}\right| Y_{2^{\nu_{1}}-1,2^{\nu_{2}-1,0}, 0}^{\theta}(f)_{p}\right)^{\frac{1}{\theta}}
$$

From (2), it follows that $H_{5}<\infty . H_{6}, H_{7}, H_{8}$ can be estimated similarly to $H_{5}$ and we have

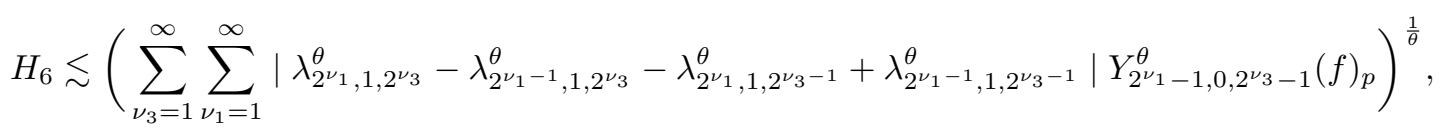

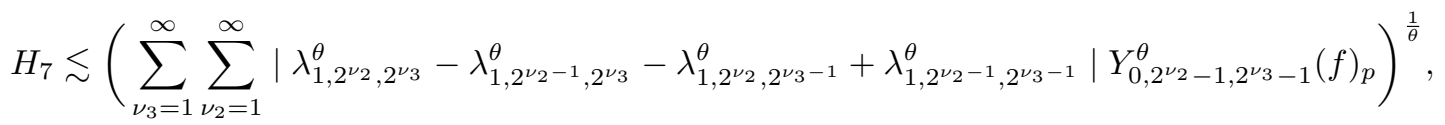

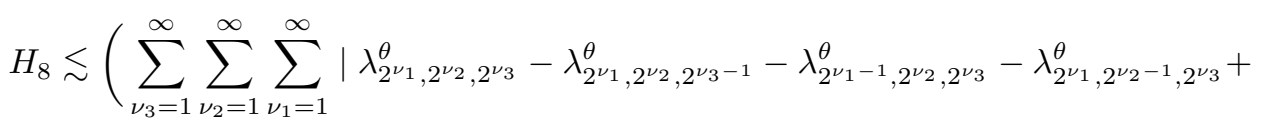

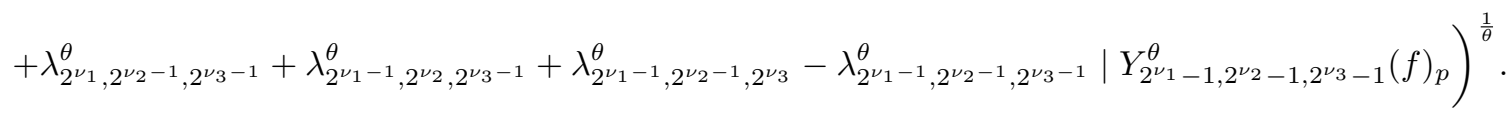

Collecting estimates of $H_{1}-H_{8}$ we get $I_{1}<\infty$. Hence, by Lemma 2.5 (b), there exists a function $g\left(x_{1}, x_{2}, x_{3}\right) \in L_{p}^{0}$, with the Fourier series

$$
\sum_{n_{1}=1}^{\infty} \sum_{n_{2}=1}^{\infty} \sum_{n_{3}=1}^{\infty} \lambda_{2^{n_{1}-1}, 2^{n_{2}-1}, 2^{n_{3}-1}} \Delta_{n_{1}, n_{2}, n_{3}}
$$

and

$$
\|g\|_{p} \leq C(p) I_{1}
$$

We rewrite series (6) in the form of $\sum_{n_{1}=1}^{\infty} \sum_{n_{2}=1}^{\infty} \sum_{n_{3}=1}^{\infty} \gamma_{n_{1}, n_{2}, n_{3}} A_{n_{1}, n_{2}, n_{3}}\left(x_{1}, x_{2}, x_{3}\right)$, where

$$
\begin{gathered}
\gamma_{1,1,1}=\lambda_{1,1,1}, \gamma_{1, \nu_{2}, \nu_{3}}=\lambda_{1,2^{n_{2}-1}, 2^{n_{3}-1}} \text { for } 2^{n_{2}-1} \leq \nu_{2} \leq 2^{n_{2}}-1,2^{n_{3}-1} \leq \nu_{3} \leq 2^{n_{3}}-1 \quad\left(n_{2}=2,3 \ldots\right) \\
\gamma_{\nu_{1}, 1,1}=\lambda_{2^{n_{1}-1}, 1,1} \text { for } 2^{n_{1}-1} \leq \nu_{1} \leq 2^{n_{1}}-1 \quad\left(n_{2}=2,3 \ldots\right) \\
\gamma_{\nu_{1}, 1, \nu_{3}}=\lambda_{2^{n_{1}-1}, 1,2^{n_{3}-1}} \text { for } 2^{n_{1}-1} \leq \nu_{1} \leq 2^{n_{1}}-1,2^{n_{3}-1} \leq \nu_{3} \leq 2^{n_{3}}-1, \quad\left(n_{1}, n_{3}=2,3 \ldots\right) \\
\gamma_{\nu_{1}, \nu_{2}, 1}=\lambda_{2^{n_{1}-1}, 2^{n_{2}-1}, 1} \text { for } 2^{n_{1}-1} \leq \nu_{1} \leq 2^{n_{1}}-1,2^{n_{2}-1} \leq \nu_{2} \leq 2^{n_{2}}-1, \quad\left(n_{1}, n_{2}=2,3 \ldots\right) \\
\gamma_{1,1, \nu_{3}}=\lambda_{1,1,2^{n_{3}-1}} \text { for } 2^{n_{3}-1} \leq \nu_{1} \leq 2^{n_{3}}-1, \quad\left(n_{3}=2,3 \ldots\right) \\
\gamma_{1, \nu_{2}, 1}=\lambda_{1,2^{n_{2}-1}, 1} \text { for } 2^{n_{2}-1} \leq \nu_{2} \leq 2^{n_{2}}-1, \quad\left(n_{2}=2,3 \ldots\right) \\
\gamma_{\nu_{1}, \nu_{2}, \nu_{3}}=\lambda_{2^{n_{1}-1}, 2^{n_{2}-1}, 2^{n_{3}-1}} \text { for } 2^{n_{1}-1} \leq \nu_{1} \leq 2^{n_{1}}-1 \\
2^{n_{2}-1} \leq \nu_{2} \leq 2^{n_{2}}-1,2^{n_{3}-1} \leq \nu_{3} \leq 2^{n_{3}}-1 \quad\left(n_{1}, n_{2}, n_{3}=2,3 \ldots\right) .
\end{gathered}
$$

Now we consider the following series

$$
\sum_{n_{1}=1}^{\infty} \sum_{n_{2}=1}^{\infty} \sum_{n_{3}=1}^{\infty} \lambda_{n_{1}, n_{2}, n_{3}} A_{n_{1}, n_{2}, n_{3}}\left(x_{1}, x_{2}, x_{3}\right)=\sum_{n_{1}=1}^{\infty} \sum_{n_{2}=1}^{\infty} \sum_{n_{3}=1}^{\infty} \gamma_{n_{1}, n_{2}, n_{3}} \Lambda_{n_{1}, n_{2}, n_{3}} A_{n_{1}, n_{2}, n_{3}}\left(x_{1}, x_{2}, x_{3}\right)
$$


where

$$
\begin{gathered}
\Lambda_{1,1,1}=1, \Lambda_{1, \nu_{2}, \nu_{3}}=\frac{\lambda_{1, \nu_{2}, \nu_{3}}}{\gamma_{1, \nu_{2}, \nu_{3}}}=\frac{\lambda_{1, \nu_{2}, \nu_{3}}}{\lambda_{1,2^{n_{2}-1}, 2^{n_{3}-1}}} \text { for } 2^{n_{2}-1} \leq \nu_{2} \leq 2^{n_{2}}-1, \\
2^{n_{3}-1} \leq \nu_{3} \leq 2^{n_{3}}-1 \quad\left(n_{2}, n_{3}=2,3 \ldots\right), \\
\Lambda_{\nu_{1}, 1,1}=\frac{\lambda_{\nu_{1}, 1,1}}{\gamma_{\nu_{1}, 1,1}}=\frac{\lambda_{\nu_{1}, 1,1}}{\lambda_{2^{n_{1}-1}, 1,1}} \text { for } 2^{n_{1}-1} \leq \nu_{1} \leq 2^{n_{1}}-1, \quad\left(n_{2}=2,3 \ldots\right), \\
\Lambda_{\nu_{1}, \nu_{2}, 1}=\frac{\lambda_{\nu_{1}, \nu_{2}, 1}}{\gamma_{\nu_{1}, \nu_{2}, 1}}=\frac{\lambda_{\nu_{1}, \nu_{2}, 1}}{\lambda_{2^{n_{1}-1}, 2^{n_{2}-1}, 1}} \text { for } 2^{n_{1}-1} \leq \nu_{1} \leq 2^{n_{1}}-1,2^{n_{2}-1} \leq \nu_{2} \leq 2^{n_{2}}-1, \quad\left(n_{1}, n_{2}=2,3 \ldots\right), \\
\Lambda_{\nu_{1}, 1, \nu_{3}}=\frac{\lambda_{\nu_{1}, 1, \nu_{3}}}{\gamma_{\nu_{1}, 1, \nu_{3}}}=\frac{\lambda_{\nu_{1}, 1, \nu_{3}}}{\lambda_{2^{n_{1}-1}, 1,2^{n_{3}-1}}} \text { for } 2^{n_{1}-1} \leq \nu_{1} \leq 2^{n_{1}}-1,2^{n_{3}-1} \leq \nu_{3} \leq 2^{n_{3}}-1, \quad\left(n_{1}, n_{2}=2,3 \ldots\right), \\
\Lambda_{1,1, \nu_{3}}=\frac{\lambda_{1,1, \nu_{3}}}{\gamma_{1,1, \nu_{3}}}=\frac{\lambda_{1,1, \nu_{3}}}{\lambda_{1,1,2^{n_{3}-1}}} \text { for } 2^{n_{3}-1} \leq \nu_{3} \leq 2^{n_{3}}-1, \\
\Lambda_{1, \nu_{2}, 1}=\frac{\lambda_{1, \nu_{2}, 1}}{\gamma_{1, \nu_{2}, 1}}=\frac{\lambda_{1, \nu_{2}, 1}}{\lambda_{1,2^{n_{2}-1}, 1}} \text { for } 2^{n_{2}-1} \leq \nu_{2} \leq 2^{n_{2}}-1, \\
\Lambda_{\nu_{1}, \nu_{2}, \nu_{3}}=\frac{\lambda_{\nu_{1}, \nu_{2}, \nu_{3}}}{\gamma_{\nu_{1}, \nu_{2}, \nu_{3}}}=\frac{\lambda_{\nu_{1}, \nu_{2}, \nu_{3}}}{\lambda_{2^{n_{1}-1}, 2^{n_{2}-1}, 2^{n_{3}-1}}} \text { for } 2^{n_{1}-1} \leq \nu_{1} \leq 2^{n_{1}}-1,2^{n_{2}-1} \leq \nu_{2} \leq 2^{n_{2}}-1, \\
2^{n_{3}-1} \leq \nu_{3} \leq 2^{n_{3}}-1 \quad\left(n_{2}, n_{3}=2,3 \ldots\right) .
\end{gathered}
$$

As shown in [6], the sequence $\left\{\Lambda_{n_{1}=1, n_{2}=1, n_{3}=1}\right\}_{n_{1}=1, n_{2}=1, n_{3}=1}^{\infty, \infty, \infty}$ satisfies the conditions of the Marcinkiewicz multiplier theorem [12], then the series (8) is the Fourier series of a function $\varphi\left(x_{1}, x_{2}, x_{2}\right) \in L_{p}$ and $\|\varphi\|_{p} \leq$ $C(\rho, \lambda)\|g\|_{p}$.

Taking into account (7) and the estimates of $H_{1}-H_{8}$ we get (3).

Let us estimate $Y_{2^{m_{1}}-1,2^{m_{2}-1}, 2^{m_{3}-1}}(\varphi)_{p}$. Using Lemma 2.4, we get

$$
\begin{gathered}
Y_{2^{m_{1}}-1,2^{m_{2}}-1,2^{m_{3}-1}}(\varphi)_{p} \leq C \| \varphi-S_{2^{m_{1}}-1, \infty, \infty}(\varphi)-S_{\infty, 2^{m_{2}-1, \infty}}(\varphi)- \\
-S_{\infty, \infty, 2^{m_{3}}-1}(\varphi)+2 S_{2^{m_{1}}-1,2^{m_{2}}-1,2^{m_{3}}-1}(\varphi) \|_{p} .
\end{gathered}
$$

We consider the series (see (8))

$$
\sum_{n_{1}=1}^{\infty} \sum_{n_{2}=1}^{\infty} \sum_{n_{3}=1}^{\infty} \lambda_{n_{1}, n_{2}, n_{3}} A_{n_{1}, n_{2}, n_{3}}^{*}\left(x_{1}, x_{2}, x_{3}\right)=\sum_{n_{1}=1}^{\infty} \sum_{n_{2}=1}^{\infty} \sum_{n_{3}=1}^{\infty} \gamma_{n_{1}, n_{2}, n_{3}} \Lambda_{n_{1}, n_{2}, n_{3}} A_{n_{1}, n_{2}, n_{3}}^{*}\left(x_{1}, x_{2}, x_{3}\right),
$$

where $A_{n_{1}, n_{2}, n_{3}}^{*}\left(x_{1}, x_{2}, x_{3}\right)=0$, if $n_{1} \leq 2^{m_{1}}-1$ and $n_{2} \leq 2^{m_{2}}-1, n_{3} \leq 2^{m_{3}}-1$ also $A_{n_{1}, n_{2}, n_{3}}^{*}\left(x_{1}, x_{2}, x_{3}\right)=$ $=A_{n_{1}, n_{2}, n_{3}}\left(x_{1}, x_{2}, x_{3}\right)$ otherwise. Since the sequence $\left\{\Lambda_{n_{1}=1, n_{2}=1, n_{3}=1}\right\}$ satisfies the conditions of the Marcinkiewicz multiplier theorem, then

$$
\left\|\sum_{n_{1}=1}^{\infty} \sum_{n_{2}=1}^{\infty} \sum_{n_{3}=1}^{\infty} \lambda_{n_{1}, n_{2}, n_{3}} A_{n_{1}, n_{2}, n_{3}}^{*}\left(x_{1}, x_{2}, x_{3}\right)\right\|_{p} \leq C\left\|\sum_{n_{1}=1}^{\infty} \sum_{n_{2}=1}^{\infty} \sum_{n_{3}=1}^{\infty} \lambda_{2^{n_{1}-1}, 2^{n_{2}-1}, 2^{n_{3}-1}} \Delta_{n_{1}, n_{2}, n_{3}}^{*}\right\|_{p},
$$

where $\Delta_{n_{1}, n_{2}, n_{3}}^{*}=0$, if $n_{1} \leq m_{1}$ and $n_{2} \leq m_{2}, n_{3} \leq m_{3} \Delta_{n_{1}, n_{2}, n_{3}}^{*}=\Delta_{n_{1} n_{2}, n_{3}}$ otherwise.

By Lemma 2.5, we have

$$
\lesssim\left\{\int_{0}^{2 \pi} \int_{0}^{2 \pi} \int_{0}^{2 \pi}\left[\sum_{k_{1}=m_{1}+1}^{\infty} \sum_{k_{2}=m_{2}+1}^{\infty} \sum_{k_{3}=m_{3}+1}^{\infty} \lambda_{2^{k_{1}-1}, 2^{k_{2}-1}, 2^{k_{3}-1}}^{2} \Delta_{k_{1}, k_{2}, k_{3}}^{2}\right]^{\frac{p}{2}} d x_{1}, d x_{2}, d x_{3}\right\}^{\frac{1}{p}} .
$$

For the sequence $\lambda_{2^{k_{1}-1}, 2^{k_{2}-1}, 2^{k_{3}-1}}$, we use inequality (5) where the index of the first element starts with $2^{m_{1}-1}, 2^{m_{2}-1}, 2^{m_{3}-1}$, and we take the sum from $2^{m_{1}-1}, 2^{m_{2}-1}, 2^{m_{3}-1}$ to $2^{k_{1}-1}, 2^{k_{2}-1}, 2^{k_{3}-1}$ respectively. The resulting inequality is substituted into inequality (9).

$$
Y_{2^{m_{1}}-1,2^{m_{2}-1}, 2^{m_{3}-1}}(\varphi)_{p} \lesssim
$$




$$
\begin{aligned}
& \lesssim\left(\lambda_{2^{m_{1}-1}, 2^{m_{2}-1}, 2^{m_{3}-1}}^{\theta}\left\{\int_{0}^{2 \pi} \int_{0}^{2 \pi} \int_{0}^{2 \pi}\left[\sum_{k_{1}=m_{1}+1}^{\infty} \sum_{k_{2}=m_{2}+1}^{\infty} \sum_{k_{3}=m_{3}+1}^{\infty} \Delta_{k_{1}, k_{2}, k_{3}}^{2}\right]^{\frac{p}{2}} d x_{1}, d x_{2}, d x_{3}\right\}^{\frac{1}{p}}+\right. \\
& +\left\{\int _ { 0 } ^ { 2 \pi } \int _ { 0 } ^ { 2 \pi } \int _ { 0 } ^ { 2 \pi } \left[\sum_{k_{1}=m_{1}+1}^{\infty} \sum_{k_{2}=m_{2}+1}^{\infty} \sum_{k_{3}=m_{3}+1}^{\infty} \Delta_{k_{1}, k_{2}, k_{3}}^{2} \times\right.\right. \\
& \left.\left.\times\left(\sum_{\nu_{1}=m_{1}+1}^{k_{1}}\left|\lambda_{2^{\nu_{1}-1}, 2^{m_{2}-1}, 2^{m_{3}-1}}^{\theta}-\lambda_{2^{\nu_{1}-2}, 2^{m_{2}-1}, 2^{m_{3}-1}}^{\theta}\right|\right)^{\frac{2}{\theta}}\right]^{\frac{p}{2}} d x_{1}, d x_{2}, d x_{3}\right\}^{\frac{1}{p}}+ \\
& +\left\{\int _ { 0 } ^ { 2 \pi } \int _ { 0 } ^ { 2 \pi } \int _ { 0 } ^ { 2 \pi } \left[\sum_{k_{1}=m_{1}+1}^{\infty} \sum_{k_{2}=m_{2}+1}^{\infty} \sum_{k_{3}=m_{3}+1}^{\infty} \Delta_{k_{1}, k_{2}, k_{3}}^{2} \times\right.\right. \\
& \left.\left.\times\left(\sum_{\nu_{2}=m_{2}+1}^{k_{2}}\left|\lambda_{2^{m_{1}-1}, 2^{\nu_{2}-1}, 2^{m_{3}-1}}^{\theta}-\lambda_{2^{m_{1}-1}, 2^{\nu_{2}-2}, 2^{m_{3}-1}}^{\theta}\right|\right)^{\frac{2}{\theta}}\right]^{\frac{p}{2}} d x_{1}, d x_{2}, d x_{3}\right\}^{\frac{1}{p}}+ \\
& +\left\{\int _ { 0 } ^ { 2 \pi } \int _ { 0 } ^ { 2 \pi } \int _ { 0 } ^ { 2 \pi } \left[\sum_{k_{1}=m_{1}+1}^{\infty} \sum_{k_{2}=m_{2}+1}^{\infty} \sum_{k_{3}=m_{3}+1}^{\infty} \Delta_{k_{1}, k_{2}, k_{3}}^{2} \times\right.\right. \\
& \left.\left.\times\left(\sum_{\nu_{3}=m_{3}+1}^{k_{3}}\left|\lambda_{2^{m_{1}-1}, 2^{m_{2}-1}, 2^{\nu_{3}-1}}^{\theta}-\lambda_{2^{m_{1}-1}, 2^{m_{2}-1}, 2^{\nu_{2}-2}}^{\theta}\right|\right)^{\frac{2}{\theta}}\right]^{\frac{p}{2}} d x_{1}, d x_{2}, d x_{3}\right\}^{\frac{1}{p}}+ \\
& +\left\{\int _ { 0 } ^ { 2 \pi } \int _ { 0 } ^ { 2 \pi } \int _ { 0 } ^ { 2 \pi } \left[\sum_{k_{1}=m_{1}+1}^{\infty} \sum_{k_{2}=m_{2}+1}^{\infty} \sum_{k_{3}=m_{3}+1}^{\infty} \Delta_{k_{1}, k_{2}, k_{3}}^{2} \times\right.\right. \\
& \times\left(\sum_{\nu_{1}=m_{1}+1}^{k_{1}} \sum_{\nu_{2}=m_{2}+1}^{k_{2}} \mid \lambda_{2^{\nu_{1}-1}, 2^{\nu_{2}-1}, 2^{m_{3}-1}}^{\theta}-\lambda_{2^{\nu_{1}-2}, 2^{\nu_{2}-1}, 2^{m_{3}-1}-}^{\theta}\right. \\
& \left.\left.\left.-\lambda_{2^{\nu_{1}-1}, 2^{\nu_{2}-2}, 2^{m_{3}-1}}^{\theta}+\lambda_{2^{\nu_{1}-2}, 2^{\nu_{2}-2}, 2^{m_{3}-1}}^{\theta} \mid\right)^{\frac{2}{\theta}}\right]^{\frac{p}{2}} d x_{1}, d x_{2}, d x_{3}\right\}^{\frac{1}{p}}+ \\
& +\left\{\int _ { 0 } ^ { 2 \pi } \int _ { 0 } ^ { 2 \pi } \int _ { 0 } ^ { 2 \pi } \left[\sum_{k_{1}=m_{1}+1}^{\infty} \sum_{k_{2}=m_{2}+1}^{\infty} \sum_{k_{3}=m_{3}+1}^{\infty} \Delta_{k_{1}, k_{2}, k_{3}}^{2} \times\right.\right. \\
& \times\left(\sum_{\nu_{2}=m_{2}+1}^{k_{2}} \sum_{\nu_{3}=m_{3}+1}^{k_{3}} \mid \lambda_{2^{m_{1}-1}, 2^{\nu_{2}-1}, 2^{\nu_{3}-1}}^{\theta}-\lambda_{2^{m_{1}-1}, 2^{\nu_{2}-2}, 2^{\nu_{3}-1}}^{\theta}-\right. \\
& \left.\left.\left.-\lambda_{2^{m_{1}-1}, 2^{\nu_{2}-1}, 2^{\nu_{3}-2}}^{\theta}+\lambda_{2^{m_{1}-1}, 2^{\nu_{2}-2}, 2^{\nu_{3}-2}}^{\theta} \mid\right)^{\frac{2}{\theta}}\right]^{\frac{p}{2}} d x_{1}, d x_{2}, d x_{3}\right\}^{\frac{1}{p}}+ \\
& +\left\{\int _ { 0 } ^ { 2 \pi } \int _ { 0 } ^ { 2 \pi } \int _ { 0 } ^ { 2 \pi } \left[\sum_{k_{1}=m_{1}+1}^{\infty} \sum_{k_{2}=m_{2}+1}^{\infty} \sum_{k_{3}=m_{3}+1}^{\infty} \Delta_{k_{1}, k_{2}, k_{3}}^{2} \times\right.\right. \\
& \times\left(\sum_{\nu_{1}=m_{1}+1}^{k_{1}} \sum_{\nu_{3}=m_{3}+1}^{k_{3}} \mid \lambda_{2^{\nu_{1}-1}, 2^{m_{2}-1}, 2^{\nu_{3}-1}}^{\theta}-\lambda_{2^{\nu_{1}-2}, 2^{m_{2}-1}, 2^{\nu_{3}-1}}^{\theta}-\right. \\
& \left.\left.\left.-\lambda_{2^{\nu_{1}-1}, 2^{m_{2}-1}, 2^{\nu_{3}-2}}^{\theta}+\lambda_{2^{\nu_{1}-2}, 2^{m_{2}-1}, 2^{\nu_{3}-2}}^{\theta} \mid\right)^{\frac{2}{\theta}}\right]^{\frac{p}{2}} d x_{1}, d x_{2}, d x_{3}\right\}^{\frac{1}{p}}+ \\
& +\left\{\int _ { 0 } ^ { 2 \pi } \int _ { 0 } ^ { 2 \pi } \int _ { 0 } ^ { 2 \pi } \left[\sum_{k_{1}=m_{1}+1}^{\infty} \sum_{k_{2}=m_{2}+1}^{\infty} \sum_{k_{3}=m_{3}+1}^{\infty} \Delta_{k_{1}, k_{2}, k_{3}}^{2} \times\right.\right. \\
& \times\left(\sum_{\nu_{1}=m_{1}+1}^{k_{1}} \sum_{\nu_{2}=m_{2}+1}^{k_{2}} \sum_{\nu_{3}=m_{3}+1}^{k_{3}} \mid \lambda_{2^{\nu_{1}-1}, 2^{\nu_{2}-1}, 2^{\nu_{3}-1}}^{\theta}-\lambda_{2^{\nu_{1}-2}, 2^{\nu_{2}-1}, 2^{\nu_{3}-1}}^{\theta}\right.
\end{aligned}
$$

Mathematics series. № 3(103)/2021 


$$
\begin{gathered}
-\lambda_{2^{\nu_{1}-1}, 2^{\nu_{2}-2}, 2^{\nu_{3}-1}}^{\theta}-\lambda_{2^{\nu_{1}-1}, 2^{\nu_{2}-1}, 2^{\nu_{3}-2}}^{\theta}+\lambda_{2^{\nu_{1}-1}, 2^{\nu_{2}-2}, 2^{\nu_{3}-2}}^{\theta}+\lambda_{2^{\nu_{1}-2}, 2^{\nu_{2}-1}, 2^{\nu_{3}-2}}^{\theta}+ \\
\left.\left.\left.\left.+\lambda_{2^{\nu_{1}-2}, 2^{\nu_{2}-2}, 2^{\nu_{3}-1}}^{\theta}-\lambda_{2^{\nu_{1}-2}, 2^{\nu_{2}-2}, 2^{\nu_{3}-2}}^{\theta} \mid\right)^{\frac{2}{\theta}}\right]^{\frac{p}{2}} d x_{1}, d x_{2}, d x_{3}\right\}^{\frac{1}{p}}\right)=: L_{1}+L_{2}+L_{3}+L_{4}+L_{5}+L_{6}+L_{7}+L_{8} .
\end{gathered}
$$

We estimate $L_{1}$ as $H_{1}$, to get

$$
\begin{aligned}
L_{1} \leq \lambda_{2^{m_{1}-1}, 2^{m_{2}-1}, 2^{m_{3}-1}}^{\theta} & \left\{\int_{0}^{2 \pi} \int_{0}^{2 \pi} \int_{0}^{2 \pi}\left[\sum_{k_{1}=m_{1}+1}^{\infty} \sum_{k_{2}=m_{2}+1}^{\infty} \sum_{k_{3}=m_{3}+1}^{\infty} \Delta_{k_{1}, k_{2}, k_{3}}^{2}\right]^{\frac{p}{2}} d x_{1}, d x_{2}, d x_{3}\right\}^{\frac{1}{p}} \lesssim \\
& \lesssim \lambda_{2^{m_{1}-1}, 2^{m_{2}-1,2^{m_{3}-1}} Y_{2^{m_{1}}-1,2^{m_{2}-1}, 2^{m_{3}-1}}(f)_{p} .}
\end{aligned}
$$

We also have

$$
\begin{aligned}
& L_{2}=\left\{\int _ { 0 } ^ { 2 \pi } \int _ { 0 } ^ { 2 \pi } \int _ { 0 } ^ { 2 \pi } \left[\sum_{k_{1}=m_{1}+1}^{\infty} \sum_{k_{2}=m_{2}+1}^{\infty} \sum_{k_{3}=m_{3}+1}^{\infty} \Delta_{k_{1}, k_{2}, k_{3}}^{2} \times\right.\right. \\
& \left.\left.\times\left(\sum_{\nu_{1}=m_{1}+1}^{k_{1}}\left|\lambda_{2^{\nu_{1}-1}, 2^{m_{2}-1}, 2^{m_{3}-1}}^{\theta}-\lambda_{2^{\nu_{1}-2}, 2^{m_{2}-1}, 2^{m_{3}-1}}^{\theta}\right|\right)^{\frac{2}{\theta}}\right]^{\frac{p}{2}} d x_{1}, d x_{2}, d x_{3}\right\}^{\frac{1}{p}} \lesssim \\
& \lesssim\left(\sum_{\nu_{1}=m_{1}+1}^{\infty}\left|\lambda_{2^{\nu_{1}-1}, 2^{m_{2}-1}, 2^{m_{3}-1}}^{\theta}-\lambda_{2^{\nu_{1}-2}, 2^{m_{2}-1}, 2^{m_{3}-1}}^{\theta}\right| \times\right. \\
& \left.\times\left\{\int_{0}^{2 \pi} \int_{0}^{2 \pi} \int_{0}^{2 \pi}\left[\sum_{k_{1}=\nu_{1}}^{\infty} \sum_{k_{2}=m_{2}+1}^{\infty} \sum_{k_{3}=m_{3}+1}^{\infty} \Delta_{k_{1}, k_{2}, k_{3}}^{2}\right]^{\frac{p}{2}} d x_{1}, d x_{2}, d x_{3}\right\}^{\frac{\theta}{p}}\right)^{\frac{1}{\theta}} \lesssim \\
& \lesssim\left(\sum_{\nu_{1}=m_{1}+1}^{\infty}\left|\lambda_{2^{\nu_{1}-1}, 2^{m_{2}-1}, 2^{m_{3}-1}}^{\theta}-\lambda_{2^{\nu_{1}-2}, 2^{m_{2}-1}, 2^{m_{3}-1}}^{\theta}\right| Y_{2^{\nu_{1}-1}-1,2^{m_{2}-1,2^{m_{3}-1}}}^{\theta}(f)_{p}\right)^{\frac{1}{\theta}} .
\end{aligned}
$$

Similarly, we obtain the estimates for $L_{3}, L_{4}$

$$
\begin{aligned}
& L_{3} \lesssim\left(\sum_{\nu_{2}=m_{2}+1}^{\infty}\left|\lambda_{2^{m_{1}-1}, 2^{\nu_{2}-1}, 2^{m_{3}-1}}^{\theta}-\lambda_{2^{m_{1}-1}, 2^{\nu_{2}-2}, 2^{m_{3}-1}}^{\theta}\right| Y_{2^{m_{1}}-1,2^{\nu_{2}-1}-1,2^{m_{3}}-1}^{\theta}(f)_{p}\right)^{\frac{1}{\theta}}, \\
& L_{4} \lesssim\left(\sum_{\nu_{3}=m_{3}+1}^{\infty}\left|\lambda_{2^{m_{1}-1}, 2^{m_{2}-1}, 2^{\nu_{3}-1}}^{\theta}-\lambda_{2^{m_{1}-1}, 2^{m_{2}-1}, 2^{\nu_{2}-2}}^{\theta}\right| Y_{2^{m_{1}}-1,2^{m_{2}-1,2^{\nu_{3}-1}-1}}^{\theta}(f)_{p}\right)^{\frac{1}{\theta}} .
\end{aligned}
$$

We estimate $L_{5}$ as follows:

$$
\begin{aligned}
& L_{5}=\left\{\int _ { 0 } ^ { 2 \pi } \int _ { 0 } ^ { 2 \pi } \int _ { 0 } ^ { 2 \pi } \left[\sum_{k_{1}=m_{1}+1}^{\infty} \sum_{k_{2}=m_{2}+1}^{\infty} \sum_{k_{3}=m_{3}+1}^{\infty} \Delta_{k_{1}, k_{2}, k_{3}}^{2} \times\left(\sum_{\nu_{1}=m_{1}+1}^{k_{1}} \sum_{\nu_{2}=m_{2}+1}^{k_{2}} \mid \lambda_{2^{\nu_{1}-1}, 2^{\nu_{2}-1}, 2^{m_{3}-1}-}^{\theta}\right.\right.\right. \\
& \left.\left.\left.-\lambda_{2^{\nu_{1}-2}, 2^{\nu_{2}-1}, 2^{m_{3}-1}}^{\theta}-\lambda_{2^{\nu_{1}-1}, 2^{\nu_{2}-2}, 2^{m_{3}-1}}^{\theta}+\lambda_{2^{\nu_{1}-2}, 2^{\nu_{2}-2}, 2^{m_{3}-1}}^{\theta} \mid\right)^{\frac{2}{\theta}}\right]^{\frac{p}{2}} d x_{1}, d x_{2}, d x_{3}\right\}^{\frac{1}{p}} \lesssim \\
& \lesssim\left(\sum_{\nu_{1}=m_{1}+1}^{\infty} \sum_{\nu_{2}=m_{2}+1}^{\infty} \mid \lambda_{2^{\nu_{1}-1}, 2^{\nu_{2}-1}, 2^{m_{3}-1}}^{\theta}-\lambda_{2^{\nu_{1}-2}, 2^{\nu_{2}-1}, 2^{m_{3}-1}}^{\theta}-\lambda_{2^{\nu_{1}-1}, 2^{\nu_{2}-2}, 2^{m_{3}-1}}^{\theta}+\right. \\
& \left.\left.+\lambda_{2^{\nu_{1}-2}, 2^{\nu_{2}-2}, 2^{m_{3}-1}}^{\theta} \mid \times \int_{0}^{2 \pi} \int_{0}^{2 \pi} \int_{0}^{2 \pi}\left[\sum_{k_{1}=\nu_{1}}^{\infty} \sum_{k_{2}=\nu_{2}}^{\infty} \sum_{k_{3}=m_{3}+1}^{\infty} \Delta_{k_{1}, k_{2}, k_{3}}^{2}\right]^{\frac{p}{2}} d x_{1}, d x_{2}, d x_{3}\right\}^{\frac{\theta}{p}}\right)^{\frac{1}{\theta}} \lesssim \\
& \lesssim\left(\sum_{\nu_{1}=m_{1}+1}^{\infty} \sum_{\nu_{2}=m_{2}+1}^{\infty} \mid \lambda_{2^{\nu_{1}-1}, 2^{\nu_{2}-1}, 2^{m_{3}-1}}^{\theta}-\lambda_{2^{\nu_{1}-2}, 2^{\nu_{2}-1}, 2^{m_{3}-1}-}^{\theta}\right. \\
& \left.-\lambda_{2^{\nu_{1}-1}, 2^{\nu_{2}-2}, 2^{m_{3}-1}}^{\theta}+\lambda_{2^{\nu_{1}-2}, 2^{\nu_{2}-2}, 2^{m_{3}-1}}^{\theta} \mid Y_{2^{\nu_{1}-1}-1,2^{\nu_{2}-1}-1,2^{m_{3}-1},}^{\theta}(f)_{p}\right)^{\frac{1}{\theta}} .
\end{aligned}
$$


Similarly, we obtain the estimates for $L_{6}, L_{7}$ :

$$
\begin{gathered}
L_{6} \lesssim\left(\sum_{\nu_{2}=m_{2}+1}^{\infty} \sum_{\nu_{3}=m_{3}+1}^{\infty} \mid \lambda_{2^{m_{1}-1}, 2^{\nu_{2}-1}, 2^{\nu_{3}-1}}^{\theta}-\lambda_{2^{m_{1}-1}, 2^{\nu_{2}-2}, 2^{\nu_{3}-1}-}^{\theta}\right. \\
\left.-\lambda_{2^{m_{1}-1}, 2^{\nu_{2}-1}, 2^{\nu_{3}-2}}^{\theta}+\lambda_{2^{m_{1}-1}, 2^{\nu_{2}-2}, 2^{\nu_{3}-2}}^{\theta} \mid Y_{2^{m_{1}}-1,2^{\nu_{2}-1}-1,2^{\nu_{3}-1}-1}^{\theta}(f)_{p}\right)^{\frac{1}{\theta}} . \\
L_{7} \lesssim\left(\sum_{\nu_{1}=m_{1}+1}^{\infty} \sum_{\nu_{3}=m_{3}+1}^{\infty} \mid \lambda_{2^{\nu_{1}-1}, 2^{m_{2}-1}, 2^{\nu_{3}-1}-\lambda_{2^{\nu_{1}-2}, 2^{m_{2}-1}, 2^{\nu_{3}-1}-}^{\theta}}^{\theta}\right. \\
\left.-\lambda_{2^{\nu_{1}-1}, 2^{m_{2}-1}, 2^{\nu_{3}-2}}^{\theta}+\lambda_{2^{\nu_{1}-2}, 2^{m_{2}-1}, 2^{\nu_{3}-2}}^{\theta} \mid Y_{2^{\nu_{1}-1}-1,2^{m_{2}}-1,2^{\nu_{3}-1}-1}^{\theta}(f)_{p}\right)^{\frac{1}{\theta}} .
\end{gathered}
$$

Finally, we estimate $L_{8}$ as follows:

$$
\begin{gathered}
L_{8} \lesssim\left(\sum_{\nu_{1}=m_{1}+1}^{\infty} \sum_{\nu_{2}=m_{2}+1}^{k_{2}} \sum_{\nu_{3}=m_{3}+1}^{\infty} \mid \lambda_{2^{\nu_{1}-1}, 2^{\nu_{2}-1}, 2^{\nu_{3}-1}}^{\theta}-\lambda_{2^{\nu_{1}-2}, 2^{\nu_{2}-1}, 2^{\nu_{3}-1}}^{\theta}-\right. \\
-\lambda_{2^{\nu_{1}-1}, 2^{\nu_{2}-2}, 2^{\nu_{3}-1}}^{\theta}-\lambda_{2^{\nu_{1}-1}, 2^{\nu_{2}-1}, 2^{\nu_{3}-2}}^{\theta}+\lambda_{2^{\nu_{1}-1}, 2^{\nu_{2}-2}, 2^{\nu_{3}-2}}^{\theta}+\lambda_{2^{\nu_{1}-2}, 2^{\nu_{2}-1}, 2^{\nu_{3}-2}}^{\theta}+ \\
\left.\quad+\lambda_{2^{\nu_{1}-2}, 2^{\nu_{2}-2}, 2^{\nu_{3}-1}}^{\theta}-\lambda_{2^{\nu_{1}-2}, 2^{\nu_{2}-2}, 2^{\nu_{3}-2}}^{\theta} \mid Y_{2^{\nu_{1}-1}-1,2^{\nu_{2}-1}-1,2^{\nu_{3}-1}-1}^{\theta}(f)_{p}\right)^{\frac{1}{\theta}} .
\end{gathered}
$$

Taking into account the estimates for $L_{1}-L_{8}$, we obtain (4). The theorem is proved.

\section{References}

1 Bernstein S. On the best approximation of continuous functions by polynomials of a given degree / S. Bernstein // Comm. Soc. Math. Kharkow, Ser. - 1912. - 2. - No. 13. - P. 49-194.

2 Devore R. Constructive Approximation / R. Devore, G.G. Lorentz. - Berlin: Springer-Verlag, 1993.

3 Steckin S.B. On best approximation of conjugate functions by trigonometric polynomials / S.B. Steckin // Izv. Akad. Nauk SSSR. - 1956. - 20. - P. 197-206.

4 Konyushkov A.A. Best approximations by trigonometric polynomials and Fourier coefficients A.A. Konyushkov // Mat. Sb.(N.S.). Is. - 1958. - 1. - Vol. 44. - No. 86. - P. 53-84.

5 Тиман М.Ф. Наилучшее приближение и модуль гладкости функций, заданных на всей вещественной оси / М.Ф. Тиман // Изв. высш. учеб. зав. Математика. - 1961. - 25. - № 6. - С. 108-120.

6 Jumabayeva A.A. Liouville-Weyl derivatives of double trigonometric series / A. Jumabayeva, B.V. Simonov // Topics in Classical and Modern Analysis. Applied and Numerical Harmonic Analysis. - 2019. - No. 1. - P. 159-182.

7 Jumabayeva A.A. Inequalities for moduli of smoothness of functions and their Liouville-Weyl derivatives / A. Jumabayeva, B.V. Simonov // Acta Math. Hungar. - 2018. - 156. - No. 1. - P. 1-17.

8 Потапов М.К. Дробные модули гладкости / М.К. Потапов, Б.В. Симонов, С.Ю. Тихонов. - М.: MAKC-Пресс, 2016. - 338 с.

9 Potapov M.K. Study of some classes of functions for help approximation by "angle" / M.K. Potapov // Tr. Steklov Mathematical Institute of the USSR. - 1972. - Vol. 117. - P. 256-291.

10 Dyachenko M. Convergence of trigonometric series with general monotone coefficients / M. Dyachenko, S. Tikhonov // C.R.Acad. Sci. Paris. - 2007. - Vol. 345. - Is. 3. - 1. - P. 123-126.

11 Tikhonov S. Trigonometric series with general monotone coefficients / S. Tikhonov // J. Math. Anal. Appl. - 2007. - 326. - P. 721-735.

12 Nikolskii S.M. Approximation of Functions of Many Variables and Embedding Theorems / S.M. Nikolskii. - M.: Nauka, 1969. 


\title{
А.Е. Жетписбаева, А.А. Жумабаева
}

Л.Н. Гумилёв атындазы Еуразия ұлттық, университеті, Нұр-Сұлтан, Қазақстан

\section{Жалпыланған Лиувилл-Вейл туындыларының бұрыштық ең жақын жуықтауларының жоғарғы бағалаулары}

\begin{abstract}
Мақалада $2 \pi$ периодты $f$ үзіліссіз функциялар және оларды тригонометриялық көпмүшеліктермен жуықтауқа жалпыланған Лиувиль-Вейл туындылары арқылы берілген үш өлшемді функциялардың бұрыштық ең жақын жуықтауын бастапқы берілген функциялардың бұрыштық ең жақын жуықтауы арқылы бағалауы қарастырылған. Авторлар классикалық Вейл аралас туындыларының орнына жалпыланған Лиувиль-Вейл туындыларын зерттеген. Қарастырылатын мәселелерді таңдағанда осы мақаладағы екінші автордың жұмысынан кейін қалыптасқан жалпы тәсілді ұстанған. Басты мақсат жұмыстың нәтижелерін үш өлшемді жағдайда дәлелдеу. Жалпы монотонды тізбектер туралы түсінік осы зерттеуде басты рөл атқарады. Функцияның ең жақын жуықтауларына қатысты $r$ туындысының ең жақын жуықтаулары, норма үшін бірнеше белгілі теңсіздіктер көрсетілген. Мақалада қарастырылған мәселелер Бернштейннің зерттелген еңбектерінің мәселелеріне жатады. Кейінірек Стечкин және Конюшков $f^{(r)}$ ең жақын жуықтау үшін теңсіздік алынды. Сонымен қатар Потаповтың еңбектерінде бұрыштарды жақындату арқылы функциялардың кейбір кластары қарастырылған. Бірінші бөлімде қажетті түсініктермен және пайдалы леммалар берілген. Үш өлшемді жағдайда жалпыланған Лиувилль-Вейл туындысы арқылы берілген функциялардың нормасының және бұрыштық ең жақын жуықтауының бағалауы алынды.
\end{abstract}

Kiлm сөздер: Лебег кеңістігі, үш өлшемді бұрышпен ең жақын жуықтау, тригонометриялық көпмүшелік, Лиувилл-Вейл туындысы.

\section{А.Е. Жепписбаева, А.А. Жумабаева}

Евразийский начионалъный университет имени Л.Н. Гумилёва, Нур-Султан, Казахстан

\section{Верхние оценки угловых наилучших приближений обобщенных производных Лиувилля-Вейля}

В статье рассмотрены непрерывные функции $f$ с периодом $2 \pi$ и их приближения тригонометрическими полиномами. Изучены оценки наилучших угловых приближений обобщены производных Лиувилля-Вейля угловым приближением функций в трехмерном случае. Авторами обобщенные производные Лиувилля-Вейля вместо классического смешанного производного Вейля. При выборе рассматриваемых вопросов они следовали общему подходу, сформировавшемуся после работы второго автора настоящей статьи. Главная цель - доказать аналоги результатов работы в трехмерном случае. Понятие общих монотонных последовательностей играет ключевую роль в исследовании. Указаны несколько известных неравенств для норм, наилучших приближений $r$-го производного по наилучшим приближениям функции $f$. Вопросы, рассмотренные в настоящей работе, относятся к кругу проблем, изученных в работах Бернштейном. Позднее Стечкин и Конюшков получили неравенство для наилучшего приближения $f^{(r)}$. Также в работах Потапова при помощи приближения углом изучены некоторые классы функций. В подразделе 1 авторами даны необходимые обозначения и полезные леммы. Получены оценки норм и наилучшие приближения обобщенного производного Лиувилля-Вейля в трехмерном случае.

Ключевые слова: пространство Лебега, наилучшее приближение трехмерным углом, тригонометрический полином, производная Лиувилля-Вейля.

\section{References}

1 Bernstein, S. (1912). On the best approximation of continuous functions by polynomials of a given degree. Comm.Soc. Math. Kharkow, Ser. 2(13), 49-194. 
2 Devore, R., \& Lorentz, G. G. (1993). Constructive Approximation. Berlin: Springer-Verlag.

3 Steckin, S.B. (1956). On best approximation of conjugate functions by trigonometric polynomials. Izv. Akad. Nauk SSSR 20, 197-206.

4 Konyushkov, A.A. (1958). Best approximations by trigonometric polynomials and Fourier coefficients. Mat. Sb.(N.S.), 1, $44(86), 53-84$.

5 Timan, M.F. (1961). Nailuchshee priblizhenie i modul gladkosti funktsii, zadannykh na vsei veshchestvennoi osi [Best approximation and modulus of smoothness of functions prescribed on the entire real axis]. Izvestiia vysshikh uchebnykh zavedenii. Matematika - Russian Mathematics (Izvestiia VUZ. Matematika), 25, 6, 108-120 [in Russian].

6 Jumabayeva, A., \& Simonov, B.V. (2019). Liouville-Weyl derivatives of double trigonometrik series. Topics in Classical and Modern Analysis. Applied and Numerical Harmonic Analysis, 1, 159-182.

7 Jumabayeva, A., \& Simonov, B.V. (2018). Inequalities for moduli of smoothness of functions and their Liouville-Weyl derivatives. Acta Math. Hungar., 156(1), 1-17.

8 Potapov, M.K., Simonov, B.V., \& Tikhonov, S.Yu. (2016). Drobnye moduli gladkosti [Fractional moduli of smoothness]. Moscow: MAKS-Press [in Russian].

9 Potapov, M.K. (1972). Study of some classes of functions for help approximation by "angle". Tr. Steklov Mathematical Institute of the USSR, 117. 256-291.

10 Dyachenko, M., \& Tikhonov, S. (2007). Convergence of trigonometric series with general monotone coefficients. C.R.Acad. Sci. Paris, 3, 345(1), 123-126.

11 Tikhonov, S. (2007). Trigonometric series with general monotone coefficients. J.Math. Anal.Appl. 326, 721-735.

12 Nikolskii, S.M. (1969). Approximation of Functions of Many Variables and Imbedding Theorems. Moscow: Nauka. 